\title{
Vibration-based estimation of beam boundary parameters
}

\author{
Hermansen, Michael Blom; Thomsen, Jon Juel
}

Published in:

Journal of Sound and Vibration

Link to article, DOI:

10.1016/j.jsv.2018.05.016

Publication date:

2018

Document Version

Peer reviewed version

Link back to DTU Orbit

Citation (APA):

Hermansen, M. B., \& Thomsen, J. J. (2018). Vibration-based estimation of beam boundary parameters. Journal of Sound and Vibration, 429, 287-304. https://doi.org/10.1016/j.jsv.2018.05.016

\section{General rights}

Copyright and moral rights for the publications made accessible in the public portal are retained by the authors and/or other copyright owners and it is a condition of accessing publications that users recognise and abide by the legal requirements associated with these rights.

- Users may download and print one copy of any publication from the public portal for the purpose of private study or research.

- You may not further distribute the material or use it for any profit-making activity or commercial gain

- You may freely distribute the URL identifying the publication in the public portal

If you believe that this document breaches copyright please contact us providing details, and we will remove access to the work immediately and investigate your claim 


\title{
Vibration-based estimation of beam boundary parameters
}

\author{
Michael Blom Hermansen and Jon Juel Thomsen* \\ Department of Mechanical Engineering, Technical University of Denmark, DK-2800 Kgs. Lyngby, Denmark \\ 1st of September 2017
}

5 Abstract

Two methods are suggested for using measured vibrations to estimate linear boundary stiffness and damping for beams, while simultaneously estimating axial tension. Estimation is performed by fitting model boundary parameters to measured modal vibration data. The methods are validated using simulated and experimental data, and shown to be accurate when boundary parameters are not extreme, i.e. representing either zero stiffness or compliance.

Keywords: beam theory, boundary conditions, tension, estimation, Rayleigh's quotient, nonlinear regression

\section{Introduction}

We suggest two methods for estimating parameters describing boundary conditions for elastic beams using measured vibrations. Theories used in this paper are derived from beam theory and modal analysis [1, 2]. The motivation is the need to estimate tension of beams from vibration data, while taking into account beam boundaries not being ideal, i.e. bolted, riveted, welded, not fully clamped etc. A specific example of the possible use of a simultaneous tension and boundary condition estimation is the problem of estimating bolt tension or tightness from vibration data [3], as bolt tension change the contact stiffness at endpoints.

Methods exist for using vibration data to estimate tension of beams [4, 5, 6, 7], but few include estimation of boundary conditions. Methods for estimating axial tension when boundary conditions are unknown exists, for example a method which uses five mode shape measurements of a single mode to estimate four mode shape coefficients and tension [8], but without estimating boundary parameters.

Boundaries have both stiffness and damping. For damping estimation with real-world beams, difficult aspects of friction such as hysteresis may be a problem, even when estimating linearized boundary damping. Hysteresis can be modeled with a linear restoring force using a Hilbert transform [9] (loss factor hysteresis), but accuracy and physical interpretations is lost.

General system identification theory for dynamical systems can be used for estimation of unknown parameters [10, 11, 12]. Many identification procedures exists, including the restoring

\footnotetext{
${ }^{*}$ Corresponding author. E-mail address: jjt@mek.dtu.dk (Jon Juel Thomsen)
} 
force surface method [13, 14], which can be used with time signals to estimate both nonlinear and linear term parameters; the field of control theory has its own estimation procedures based on minimal noise principles [15], designed to be real time during measurements.

Linear and nonlinear parameter estimation theory [16] is relevant for estimating boundary parameters. Estimation of beam parameters using least squares minimization with experimental data has been demonstrated for specific cases [17, 18], but not for arbitrary boundaries.

A method for simultaneous estimation of axial tension and boundary parameters of beams is needed. In this work we suggest two methods: Method 1 fits measured natural frequencies, damping coefficients and mode shapes to Rayleigh's quotient using linear regression, while Method 2 fits measured natural frequencies and/or mode shapes to the solution of an eigenvalue problem using nonlinear regression. Method 2 estimate boundary stiffness parameters and tension, while Method 1 also estimates boundary damping parameters, but requires mode shapes.

Section 2 presents the beam boundary model used for analysis. Section 3 presents Method 1 and Section 4 presents Method 2. Section 5 validates the two methods using simulated and experimental data. Section 6 concludes on the validity of proposed estimation methods.

\section{Mathematical model}

Figure 1 shows a beam in tension, with linear and rotational springs [19] and dampers at its boundaries. The beam has length $l$, and axially uniform mass density $\rho$, Young's modulus $E$, area moment of inertia $I$ and cross-section area $A$. The beam performs transverse vibrations $U(X, t)$ in the plane of the paper, where $X \in[0 ; l]$ is the axial coordinate, and $t$ the time; it's curvature can be approximated as the second derivative $U^{\prime \prime}(X, t)$, and the axial tension $N$ is axially uniform and constant. Transverse and longitudinal boundary conditions consists of transverse and rotational springs $K_{1-4}$, dampers $C_{1-4}$, and prescribed axial tension $N$.

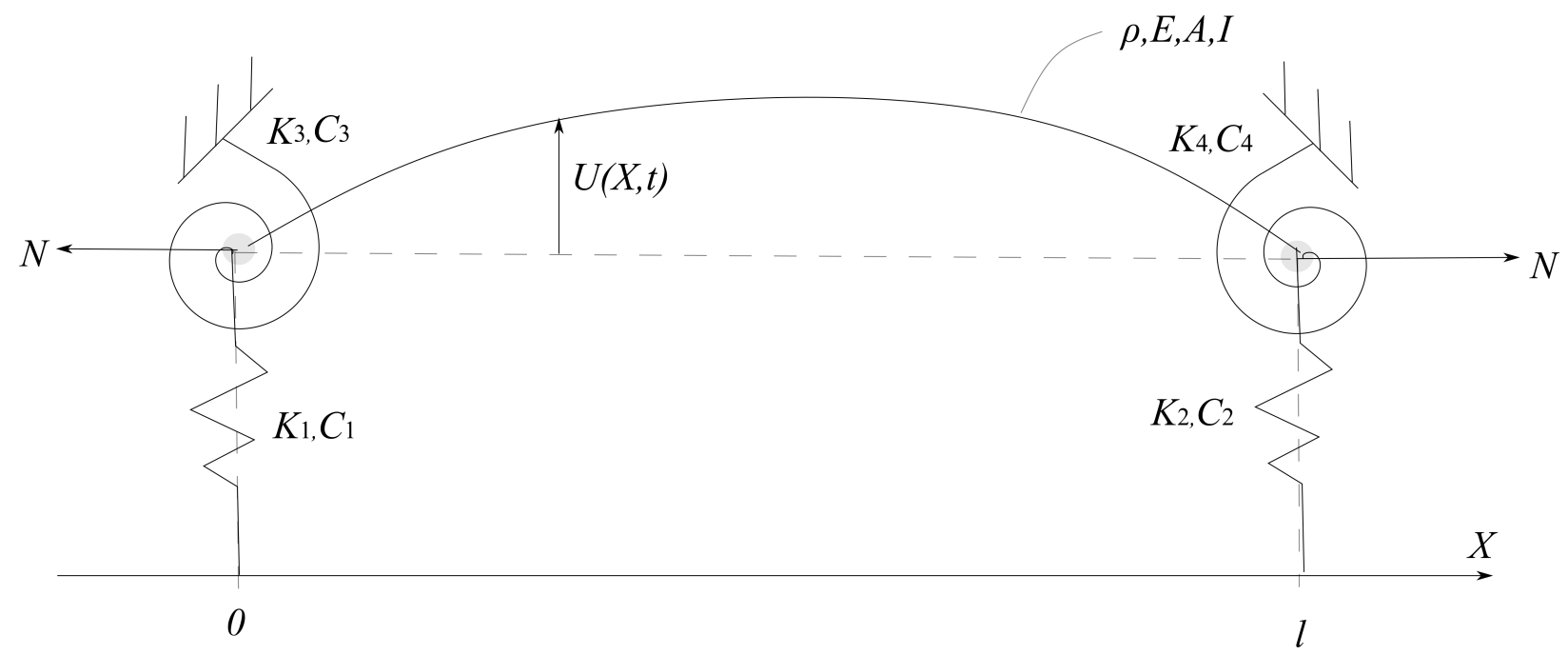

Fig. 1. Beam in tension with springs and dampers at boundaries 


\subsection{Equation of motion}

Nondimensional quantities are introduced:

$$
\begin{gathered}
x=\frac{X}{l}, u(x, \tau)=\frac{U(X, t)}{l}, \tau=\omega_{0} t, \omega_{0}=\sqrt{\frac{E I}{\rho A l^{4}}}, \\
p=\frac{N l^{2}}{E I}, k_{1}=\frac{K_{1} l^{3}}{E I}, k_{2}=\frac{K_{2} l^{3}}{E I}, k_{3}=\frac{K_{3} l}{E I}, k_{4}=\frac{K_{4} l}{E I}, \\
c_{1}=\frac{C_{1} l^{3} \omega_{0}}{E I}, c_{2}=\frac{C_{2} l^{3} \omega_{0}}{E I}, c_{3}=\frac{C_{3} l \omega_{0}}{E I}, c_{4}=\frac{C_{4} l \omega_{0}}{E I} .
\end{gathered}
$$

Nondimensional position and time are $x=X / l$ and $\tau=\omega_{0} t$, where $\omega_{0}$ is a characteristic angular frequency. The nondimensional deflection is $u(x, \tau)$ and its derivatives will be denoted $u^{\prime}$ w.r.t. $x$ and $\dot{u}$ w.r.t. $\tau$. The nondimensional tension $p$ describes a ratio between transverse beam stiffness originating from tension and from bending, and includes information about beam slenderness, since $l^{2} / I=s_{\mathrm{r}}^{2} / A$, where $s_{\mathrm{r}}=l / \sqrt{I / A}$ is the slenderness ratio. Boundary springs and dampers are nondimensionalized into $k_{1-4}$ and $c_{1-4}$.

Bernoulli-Euler's equation of motion for deflections $u(x, \tau)[1]$ is, in nondimensional form

$$
\ddot{u}+u^{\prime \prime \prime \prime}-p u^{\prime \prime}+\mathrm{D} \dot{u}=0,
$$

with boundary conditions

$$
\begin{aligned}
-u^{\prime \prime \prime}(0, \tau)+p u^{\prime}(0, \tau) & =k_{1} u(0, \tau)+c_{1} \dot{u}(0, \tau), \\
u^{\prime \prime}(0, \tau) & =k_{3} u^{\prime}(0, \tau)+c_{3} \dot{u}^{\prime}(0, \tau), \\
-u^{\prime \prime \prime}(1, \tau)+p u^{\prime}(1, \tau) & =-k_{2} u(1, \tau)-c_{2} \dot{u}(1, \tau), \\
u^{\prime \prime}(1, \tau) & =-k_{4} u^{\prime}(1, \tau)-c_{4} \dot{u}^{\prime}(1, \tau) .
\end{aligned}
$$

The equation of motion (2) has an inertial term, a bending stiffness term and a stiffness term originating from tension $p$, and a distributed viscous beam damping term $\mathrm{D} \dot{u}$, where $\mathrm{D}$ is any linear spatial differential operator needed to describe viscous beam damping. Next, we investigate how natural frequencies, mode shapes and damping coefficients depend on boundary parameters $k_{1-4}, c_{1-4}$ and tension $p$.

\subsection{Damped eigenvalue problem}

The linear equations (2)-(6) are solved by superposition of eigen-solutions

$$
u(x, \tau)=\phi(x) \mathrm{e}^{\lambda \tau},
$$

which inserted into (2)-(6) gives

$$
\begin{aligned}
\lambda^{2} \phi+\phi^{\prime \prime \prime \prime}-p \phi^{\prime \prime}+\lambda \mathrm{D} \phi & =0, \\
-\phi^{\prime \prime \prime}(0)+p \phi^{\prime}(0) & =k_{1} \phi(0)+\lambda c_{1} \phi(0), \\
\phi^{\prime \prime}(0) & =k_{3} \phi^{\prime}(0)+\lambda c_{3} \phi^{\prime}(0), \\
-\phi^{\prime \prime \prime}(1)+p \phi^{\prime}(1) & =-k_{2} \phi(1)-\lambda c_{2} \phi(1), \\
\phi^{\prime \prime}(1) & =-k_{4} \phi^{\prime}(1)-\lambda c_{4} \phi^{\prime}(1),
\end{aligned}
$$


to be solved for mode shapes $\phi(x)$ and eigenvalues $\lambda$. Undamped natural frequencies $\omega$ and damping ratios $\zeta$ are related to $\lambda$ by [2]

$$
\lambda=\omega\left(-\zeta+\mathrm{i} \sqrt{1-\zeta^{2}}\right)
$$

where in this work under-damped vibrations, $\zeta<1$, are assumed.

Convenient relationships between modal properties and boundary parameters, to be used for model fitting, will be derived. Equation (8) is multiplied by the complex conjugate mode shape $\bar{\phi}(x)$, integrated over the beams length [0;1], and $\lambda^{2}$ is solved for, giving

$$
\lambda^{2}=\frac{-\int_{0}^{1} \bar{\phi}\left(\phi^{\prime \prime \prime \prime}-p \phi^{\prime \prime}+\lambda \mathrm{D} \phi\right) d x}{\int_{0}^{1}|\phi|^{2} d x}
$$

which is a Rayleigh quotient. Partially integrating the integral in the numerator with boundary terms replaceable by (9)-(12), gives

$$
\begin{array}{r}
\lambda^{2}=-\frac{\int_{0}^{1}\left(\left|\phi^{\prime \prime}\right|^{2}+p\left|\phi^{\prime}\right|^{2}\right) d x+k_{1}|\phi(0)|^{2}+k_{2}|\phi(1)|^{2}+k_{3}\left|\phi^{\prime}(0)\right|^{2}+k_{4}\left|\phi^{\prime}(1)\right|^{2}}{\int_{0}^{1}|\phi|^{2} d x} \\
-\lambda\left(\frac{\int_{0}^{1} \bar{\phi} \mathrm{D} \phi d x+c_{1}|\phi(0)|^{2}+c_{2}|\phi(1)|^{2}+c_{3}\left|\phi^{\prime}(0)\right|^{2}+c_{4}\left|\phi^{\prime}(1)\right|^{2}}{\int_{0}^{1}|\phi|^{2} d x}\right) .
\end{array}
$$

To arrive at simple expressions for $\omega$ and $\zeta$, it will be assumed that $\int_{0}^{1} \bar{\phi} \mathrm{D} \phi d x$ is real. To justify this assumption, a damping model with viscous air damping and material damping,

$$
\mathrm{D}=c_{\mathrm{a}}+c_{\mathrm{s}}\left(\frac{\partial^{4}}{\partial x^{4}}-p \frac{\partial^{2}}{\partial x^{2}}\right)
$$

is used, where $c_{a}$ is coefficient for viscous air damping and $c_{s}$ is a coefficient for viscous material damping. In that case the integral can be shown with partial integration and boundary conditions (9)-(12) to be

$$
\begin{array}{r}
\int_{0}^{1} \bar{\phi} \mathrm{D} \phi d x=c_{\mathrm{s}}\left(\int_{0}^{1}\left(\left|\phi^{\prime \prime}\right|^{2}+p\left|\phi^{\prime}\right|^{2}\right) d x+k_{1}|\phi(0)|^{2}+k_{2}|\phi(1)|^{2}+k_{3}\left|\phi^{\prime}(0)\right|^{2}+k_{4}\left|\phi^{\prime}(1)\right|^{2}\right) \\
+c_{\mathrm{a}} \int_{0}^{1}|\phi|^{2} d x
\end{array}
$$

which is real. It is hypothesized that the integral $\int_{0}^{1} \bar{\phi} \mathrm{D} \phi d x$ will be real for dissipative viscous damping models, and have imaginary parts for damping models with gyroscopic forces, such as $\mathrm{D}=\partial / \partial x$, which can occur for e.g. beams having fluid flows within [1].

Multiplying 15 by the complex conjugate $\bar{\lambda}$, inserting $(13)$, taking imaginary and real parts (assuming $\int_{0}^{1} \bar{\phi} \mathrm{D} \phi d x$ is real), inserting $(17)$, and rearranging gives

$$
\omega^{2}=\frac{\int_{0}^{1}\left(\left|\phi^{\prime \prime}\right|^{2}+p\left|\phi^{\prime}\right|^{2}\right) d x+k_{1}|\phi(0)|^{2}+k_{2}|\phi(1)|^{2}+k_{3}\left|\phi^{\prime}(0)\right|^{2}+k_{4}\left|\phi^{\prime}(1)\right|^{2}}{\int_{0}^{1}|\phi|^{2} d x}
$$


and

$$
2 \omega \zeta=c_{\mathrm{a}}+c_{\mathrm{s}} \omega^{2}+\frac{c_{1}|\phi(0)|^{2}+c_{2}|\phi(1)|^{2}+c_{3}\left|\phi^{\prime}(0)\right|^{2}+c_{4}\left|\phi^{\prime}(1)\right|^{2}}{\int_{0}^{1}|\phi|^{2} d x},
$$

from which appears that natural frequencies squared, and damping coefficients $2 \omega \zeta$ are linear functions of boundary parameters $k_{1-4}, c_{1-4}$, tension $p$, and damping beam damping parameters $c_{\mathrm{a}}$ and $c_{\mathrm{s}}$. Given measured mode shapes, natural frequencies and damping ratios, (18)-(19) can be used to estimate boundary parameters and tension through linear regression, cf. Section 3 . Distributed beam damping parameters $c_{\mathrm{a}}$ and $c_{\mathrm{s}}$ can be included in the regression, as these appear linearly in (19).

Calculating undamped natural frequencies and damping coefficients using Rayleigh's quotients has been done for proportionally damped multi-degree of freedom systems [20], but proportional damping is not required or assumed for (19).

\subsection{Undamped eigenvalue problem}

For engineering structures, damping forces are typically weak compared to elastic and inertia forces, and affects only negligibly natural frequencies and mode shapes, which are considered functions of boundary stiffness and tension only. In that case $\lambda=i \omega$ and an undamped mode shape can be solved from (8) as [8]

$$
\phi(x)=\lambda(x)^{\mathrm{T}} \mathbf{q},
$$

where $\lambda(x)=\left\{\begin{array}{llll}\cos \left(\alpha_{1} x\right) & \sin \left(\alpha_{1} x\right) & \cosh \left(\alpha_{2} x\right) & \sinh \left(\alpha_{2} x\right)\end{array}\right\}^{\mathrm{T}}, \mathbf{q} \in \mathbb{R}^{4}$ are coefficients determined by boundary conditions, and $\alpha_{1-2}$ are [8]

$$
\alpha_{1}=\sqrt{-\frac{p}{2}+\sqrt{\left(\frac{p}{2}\right)^{2}+\omega^{2}}}, \quad \alpha_{2}=\sqrt{\frac{p}{2}+\sqrt{\left(\frac{p}{2}\right)^{2}+\omega^{2}}}
$$

Inserting 20 and the identity $p=\alpha_{2}^{2}-\alpha_{1}^{2}$ into boundary conditions 9 , 12 gives:

$$
\mathbf{S q}=\mathbf{0},
$$

in which

$$
\mathbf{S}=\left[\begin{array}{cccc}
-k_{1} & \alpha_{1} \alpha_{2}^{2} & -k_{1} & -\alpha_{1}^{2} \alpha_{2} \\
k_{2} \tilde{c}_{1}-\alpha_{1} \alpha_{2}^{2} \tilde{s}_{1} & k_{2} \tilde{s}_{1}+\alpha_{1} \alpha_{2}^{2} \tilde{c}_{1} & k_{2} \tilde{c}_{2}-\alpha_{1}^{2} \alpha_{2} \tilde{s}_{2} & k_{2} \tilde{s}_{2}-\alpha_{1}^{2} \alpha_{2} \tilde{c}_{2} \\
-\alpha_{1}^{2} & -k_{3} \alpha_{1} & \alpha_{2}^{2} & -k_{3} \alpha_{2} \\
-\alpha_{1}^{2} \tilde{c}_{1}-k_{4} \alpha_{1} \tilde{s}_{1} & -\alpha_{1}^{2} \tilde{s}_{1}+k_{4} \alpha_{1} \tilde{c}_{1} & \alpha_{2}^{2} \tilde{c}_{2}+k_{4} \alpha_{2} \tilde{s}_{2} & \alpha_{2}^{2} \tilde{s}_{2}+k_{4} \alpha_{2} \tilde{c}_{2}
\end{array}\right],
$$

where

$$
\tilde{s}_{1}=\sin \alpha_{1}, \quad \tilde{s}_{2}=\sinh \alpha_{2}, \quad \tilde{c}_{1}=\cos \alpha_{1}, \quad \tilde{c}_{2}=\cosh \alpha_{2} .
$$

Condition (22) defines $\mathbf{q}$ as the eigenvector of $\mathbf{S}$, requiring zero determinant:

$$
\|\mathbf{S}\|=\mathbf{k}^{\mathrm{T}} \mathbf{A} \mathbf{a}=0
$$


where

$$
\mathbf{k}=\left\{\begin{array}{c}
1 \\
k_{1}+k_{2} \\
k_{3}+k_{4} \\
k_{1} k_{2} \\
k_{3} k_{4} \\
k_{1} k_{3}+k_{2} k_{4} \\
k_{1} k_{4}+k_{2} k_{3} \\
k_{1} k_{2}\left(k_{3}+k_{4}\right) \\
k_{3} k_{4}\left(k_{1}+k_{2}\right) \\
k_{1} k_{2} k_{3} k_{4}
\end{array}\right\}, \quad \mathbf{a}=\left\{\begin{array}{c}
1 \\
\sin \alpha_{1} \cosh \alpha_{2} \\
\sin \alpha_{1} \sinh \alpha_{2} \\
\cos \alpha_{1} \cosh \alpha_{2} \\
\cos \alpha_{1} \sinh \alpha_{2}
\end{array}\right\}
$$

and

$$
\mathbf{A}=\left[\begin{array}{ccccc}
-2 \alpha_{1}^{5} \alpha_{2}^{5} & 0 & -\alpha_{2}^{2} \alpha_{1}^{2}\left(\alpha_{1}^{6}-\alpha_{2}^{6}\right) & 2 \alpha_{1}^{5} \alpha_{2}^{5} & 0 \\
0 & \alpha_{1}^{4} \alpha_{2}\left(\alpha_{1}^{2}+\alpha_{2}^{2}\right) & 0 & 0 & -\alpha_{2}^{4} \alpha_{1}\left(\alpha_{1}^{2}+\alpha_{2}^{2}\right) \\
0 & \alpha_{1}^{2} \alpha_{2}^{5}\left(\alpha_{1}^{2}+\alpha_{2}^{2}\right) & 0 & 0 & \alpha_{1}^{5} \alpha_{2}^{2}\left(\alpha_{1}^{2}+\alpha_{2}^{2}\right) \\
0 & 0 & -\left(\alpha_{1}^{2}+\alpha_{2}^{2}\right)^{2} & 0 & 0 \\
0 & 0 & \alpha_{1}^{2} \alpha_{2}^{2}\left(\alpha_{1}^{2}+\alpha_{2}^{2}\right)^{2} & 0 & 0 \\
-2 \alpha_{1}^{3} \alpha_{2}^{3} & 0 & \alpha_{1}^{2} \alpha_{2}^{2}\left(\alpha_{1}^{2}-\alpha_{2}^{2}\right) & -\alpha_{1} \alpha_{2}\left(\alpha_{1}^{4}+\alpha_{2}^{4}\right) & 0 \\
0 & 0 & 0 & -\alpha_{1} \alpha_{2}\left(\alpha_{1}^{2}+\alpha_{2}^{2}\right)^{2} & 0 \\
0 & -\alpha_{2}\left(\alpha_{1}^{2}+\alpha_{2}^{2}\right) & 0 & 0 & \alpha_{1}\left(\alpha_{1}^{2}+\alpha_{2}^{2}\right) \\
0 & -\alpha_{1}^{2} \alpha_{2}\left(\alpha_{1}^{2}+\alpha_{2}^{2}\right) & 0 & 0 & -\alpha_{2}^{2} \alpha_{1}\left(\alpha_{1}^{2}+\alpha_{2}^{2}\right) \\
-2 \alpha_{1} \alpha_{2} & 0 & \alpha_{1}^{2}-\alpha_{2}^{2} & 2 \alpha_{1} \alpha_{2} & 0
\end{array}\right] .
$$

Hyperbolic terms in a are normalized by multiplying (25) with $e^{-\alpha_{2}}$, which gives

$$
\mathbf{k}^{\mathrm{T}} \mathbf{r}=0
$$

where

$$
\mathbf{r}=\mathbf{A} \mathbf{a} e^{-\alpha_{2}} .
$$

Since $\mathbf{r}$ is a function of $\alpha_{1}$ and $\alpha_{2}$, which are functions of natural frequency $\omega$ and tension $p$ in (21), (28) implicitly gives natural frequency $\omega$ as a function of tension $p$ and boundary stiffness parameters in k. Newton-Raphson iteration can be used to solve (28) for $\omega$ 's with initial values obtained from a zero-crossing sweep.

\subsection{Relationship between boundary stiffness and natural frequencies}

To illustrate how boundary stiffness affect natural frequencies, a beam with parameters $p=$ $0, k_{1} \rightarrow \infty, k_{3} \rightarrow \infty, k_{4}=0$ and a finite transverse stiffness $k_{2}$ at its right end is considered. Figure 2 (a) shows the relationship between $\omega$ and boundary stiffness $k_{2}$ for the eight lowest modes, showing natural frequencies being insensitive to changes in very small or very large boundary stiffness. This means that very large or very small boundary stiffness cannot be estimated accurately from 

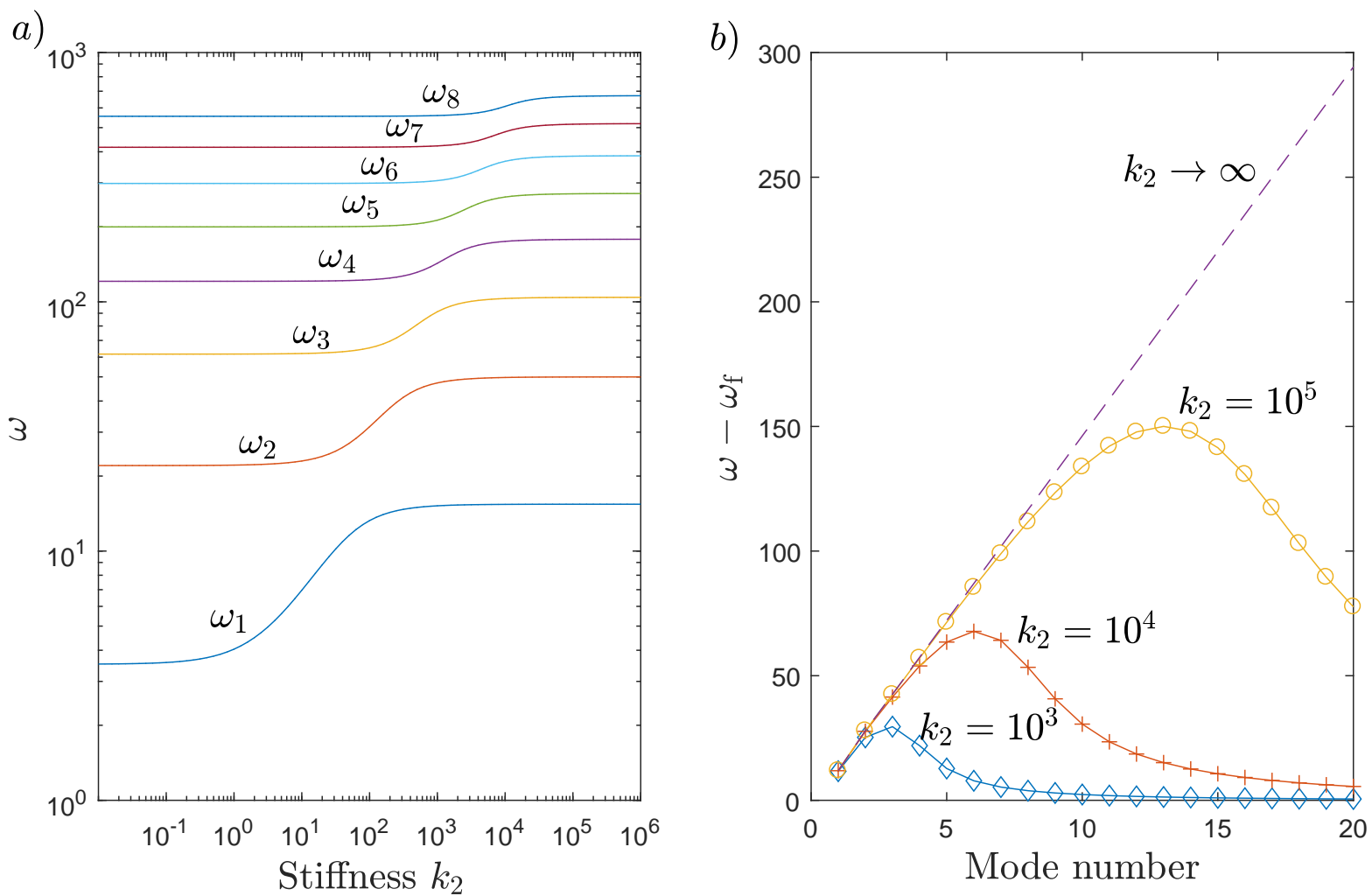

Fig. 2. a) Natural frequencies $\omega_{1-8}$ versus boundary stiffness $k_{2}$ and b) difference $\omega-\omega_{\mathrm{f}}$ between real and clamped-free natural frequencies as functions of mode number for varying $k_{2}$ and $p=k_{4}=0, k_{1}=k_{3} \rightarrow \infty$

natural frequencies. The beam vibrates as a clamped-hinged beam $\left(k_{2} \rightarrow \infty\right)$ when $k_{2}$ is large, and as a clamped-free beam $\left(k_{2}=0\right)$ when $k_{2}$ is small, in between having a transition occurring at higher stiffness $k_{2}$ for higher modes. Figure 2 (b) further illustrates how natural frequencies change as if the right boundary with $k_{2}$ is hinged for the first couple of modes, and free with natural frequencies $\omega \approx \omega_{\mathrm{f}}$ for higher order modes. To estimate boundary stiffness parameters accurately, natural frequencies from modes vibrating as if boundaries are neither infinite or zero must be used.

\subsection{Theory used for estimation}

Given measured natural frequencies, damping coefficients and continuous mode shapes, (18)(19) can be used to fit boundary parameters $k_{1-4}, c_{1-4}$ and tension $p$ with linear regression, cf. Section 3. Continuous mode shapes are typically not measurable, but must be fitted to discrete mode shape measurements or estimated somehow. If damping is negligible, (28) and/or (20)-(22) can be used to fit boundary stiffness and tension to measured natural frequencies and/or mode shapes using nonlinear regression, cf. Section 4 .

Next, Sections 3 and 4 presents Method 1 and 2, while Section 5 presents examples. 


\section{Method 1: Boundary parameter estimation using Rayleigh's quotients}

It is convenient to write (18)-(19) with $c_{\mathrm{a}}=c_{\mathrm{s}}=0$ for $n$ modes in vector form,

$$
\begin{aligned}
\boldsymbol{\omega}^{2} & =\boldsymbol{\omega}_{0}^{2}+\mathbf{B}_{\mathrm{s}} \mathbf{p}_{\mathrm{s}}, \\
\mathbf{d} & =\mathbf{B}_{\mathrm{c}} \mathbf{p}_{\mathrm{c}},
\end{aligned}
$$

where $\mathbf{p}_{\mathrm{s}} \in \mathbb{R}^{5}$ and $\mathbf{p}_{\mathrm{c}} \in \mathbb{R}^{4}$ contains boundary parameters and tension:

$$
\mathbf{p}_{\mathrm{s}}=\left\{\begin{array}{lllll}
p & k_{1} & k_{2} & k_{3} & k_{4}
\end{array}\right\}^{\mathrm{T}}, \quad \mathbf{p}_{\mathrm{c}}=\left\{\begin{array}{llll}
c_{1} & c_{2} & c_{3} & c_{4}
\end{array}\right\}^{\mathrm{T}},
$$

$\boldsymbol{\omega}^{2} \in \mathbb{R}^{n}$ and $\mathbf{d} \in \mathbb{R}^{n}$ contains natural frequency squared and damping coefficients, respectively:

$$
\boldsymbol{\omega}^{2}=\left\{\begin{array}{llll}
\omega_{1}^{2} & \omega_{2}^{2} & \ldots & \omega_{n}^{2}
\end{array}\right\}^{\mathrm{T}}, \quad \mathbf{d}=\left\{\begin{array}{llll}
2 \omega_{1} \zeta_{1} & 2 \omega_{2} \zeta_{2} & \ldots & 2 \omega_{n} \zeta_{n}
\end{array}\right\}^{\mathrm{T}},
$$

and matrices $\mathbf{B}_{\mathrm{s}} \in \mathbb{R}^{n \times 5}, \mathbf{B}_{\mathrm{c}} \in \mathbb{R}^{n \times 4}$, and vector $\boldsymbol{\omega}_{0}^{2} \in \mathbb{R}^{n}$ contains mode shape elements:

$$
\begin{aligned}
\mathbf{B}_{\mathrm{s}} & =\left[\begin{array}{ccccc}
\frac{\int_{0}^{1}\left|\phi_{1}^{\prime}\right|^{2} d x}{\int_{0}^{1}\left|\phi_{1}\right|^{2} d x} & \frac{\left|\phi_{1}(0)\right|^{2}}{\int_{0}^{1}\left|\phi_{1}\right|^{2} d x} & \frac{\left|\phi_{1}(1)\right|^{2}}{\int_{0}^{1}\left|\phi_{1}\right|^{2} d x} & \frac{\left|\phi_{1}^{\prime}(0)\right|^{2}}{\int_{0}^{1}\left|\phi_{1}\right|^{2} d x} & \frac{\left|\phi_{1}^{\prime}(1)\right|^{2}}{\int_{0}^{1}\left|\phi_{1}\right|^{2} d x} \\
\ldots & \ldots & \ldots & \ldots & \ldots \\
\frac{\int_{0}^{1}\left|\phi_{n}^{\prime}\right|^{2} d x}{\int_{0}^{1}\left|\phi_{n}\right|^{2} d x} & \frac{\left|\phi_{n}(0)\right|^{2}}{\int_{0}^{1}\left|\phi_{n}\right|^{2} d x} & \frac{\left|\phi_{n}(1)^{2}\right|}{\int_{0}^{1}\left|\phi_{n}\right|^{2} d x} & \frac{\left|\phi_{n}^{\prime}(0)^{2}\right|}{\int_{0}^{1}\left|\phi_{n}\right|^{2} d x} & \frac{\left|\phi_{n}^{\prime}(1)^{2}\right|}{\int_{0}^{1}\left|\phi_{n}\right|^{2} d x}
\end{array}\right], \quad \boldsymbol{\omega}_{0}^{2}=\left\{\begin{array}{c}
\frac{\int_{0}^{1}\left|\phi_{1}^{\prime \prime}\right|^{2} d x}{\int_{0}^{1}\left|\phi_{1}\right|^{2} d x} \\
\ldots \\
\frac{\int_{0}^{1}\left|\phi_{n}^{\prime \prime}\right|^{2} d x}{\int_{0}^{1}\left|\phi_{n}\right|^{2} d x}
\end{array}\right\}, \\
\mathbf{B}_{\mathrm{c}}= & {\left[\begin{array}{cccc}
\frac{\left|\phi_{1}(0)\right|^{2}}{\int_{0}^{1}\left|\phi_{1}\right|^{2} d x} & \frac{\left|\phi_{1}(1)\right|^{2}}{\int_{0}^{1}\left|\phi_{1}\right|^{2} d x} & \frac{\left|\phi_{1}^{\prime}(0)\right|^{2}}{\int_{0}^{1}\left|\phi_{1}\right|^{2} d x} & \frac{\left|\phi_{1}^{\prime}(1)\right|^{2}}{\int_{0}^{1}\left|\phi_{1}\right|^{2} d x} \\
\ldots & \ldots & \ldots & \ldots \\
\frac{\left|\phi_{n}(0)\right|^{2}}{\int_{0}^{1}\left|\phi_{n}\right|^{2} d x} & \frac{\left|\phi_{n}(1)\right|^{2}}{\int_{0}^{1}\left|\phi_{n}\right|^{2} d x} & \frac{\left|\phi_{n}^{\prime}(0)\right|^{2}}{\int_{0}^{1}\left|\phi_{n}\right|^{2} d x} & \frac{\left|\phi_{n}^{\prime}(1)\right|^{2}}{\int_{0}^{1}\left|\phi_{n}\right|^{2} d x}
\end{array}\right] . }
\end{aligned}
$$

Beam damping have been neglected in (31), as the topic of this paper is the estimation of boundary parameters and tension.

The measurable quantities $\boldsymbol{\omega}^{2}$ and $\mathbf{d}$ are linear in the unknown parameters in $(30)-(31)$, thus linear regression can be used to estimate $\mathbf{p}_{\mathrm{s}}$ and $\mathbf{p}_{\mathrm{c}}$. Object functions describing deviations between measured and modeled data are defined [16]:

$$
\begin{aligned}
& S_{\mathrm{s}}\left(\hat{\mathbf{p}}_{\mathrm{s}}\right)=\left(\boldsymbol{\omega}^{2}-\hat{\boldsymbol{\omega}}^{2}\left(\hat{\mathbf{p}}_{\mathrm{s}}\right)\right)^{\mathrm{T}} \mathbf{W}_{\mathrm{s}}\left(\boldsymbol{\omega}^{2}-\hat{\boldsymbol{\omega}}^{2}\left(\hat{\mathbf{p}}_{\mathrm{s}}\right)\right)+\hat{\mathbf{p}}_{\mathrm{s}}^{\mathrm{T}} \mathbf{U}_{\mathrm{s}} \hat{\mathbf{p}}_{\mathrm{s}}, \\
& S_{\mathrm{c}}\left(\hat{\mathbf{p}}_{\mathrm{c}}\right)=\left(\mathbf{d}-\hat{\mathbf{d}}\left(\hat{\mathbf{p}}_{\mathrm{c}}\right)\right)^{\mathrm{T}} \mathbf{W}_{\mathrm{c}}\left(\mathbf{d}-\hat{\mathbf{d}}\left(\hat{\mathbf{p}}_{\mathrm{c}}\right)\right)+\hat{\mathbf{p}}_{\mathrm{c}}^{\mathrm{T}} \mathbf{U}_{\mathrm{c}} \hat{\mathbf{p}}_{\mathrm{c}},
\end{aligned}
$$

where $\boldsymbol{\omega}^{2}$ and $\mathbf{d}$ are measured natural frequencies and damping coefficients, and $\hat{\boldsymbol{\omega}}^{2}$ and $\hat{\mathbf{d}}$ are those predicted through (30)-(31) by $\hat{\mathbf{p}}_{\mathrm{s}}$ and $\hat{\mathbf{p}}_{\mathrm{c}}$. Matrices $\mathbf{W}_{\mathrm{s}}$ and $\mathbf{W}_{\mathrm{c}}$ weighs deviations depending on the confidence in each measurement. Weights $\mathbf{U}_{\mathrm{s}}$ and $\mathbf{U}_{\mathrm{c}}$ are used for imposing conditions on parameters if they are non-identifiable (cf. end of section), but can otherwise be set to zero. Optimal estimates can be found by minimizing $S_{\mathrm{s}}$ and $S_{\mathrm{c}}$, which gives [16]:

$$
\begin{aligned}
& \hat{\mathbf{p}}_{\mathrm{s}}=\left(\mathbf{B}_{\mathrm{s}}^{\mathrm{T}} \mathbf{W}_{\mathrm{s}} \mathbf{B}_{\mathrm{s}}+\mathbf{U}_{\mathrm{s}}\right)^{-1}\left(\mathbf{B}_{\mathrm{s}}^{\mathrm{T}} \mathbf{W}_{\mathrm{s}}\left(\boldsymbol{\omega}^{2}-\boldsymbol{\omega}_{0}^{2}\right)\right), \\
& \hat{\mathbf{p}}_{\mathrm{c}}=\left(\mathbf{B}_{\mathrm{c}}^{\mathrm{T}} \mathbf{W}_{\mathrm{c}} \mathbf{B}_{\mathrm{c}}^{\mathrm{T}}+\mathbf{U}_{\mathrm{c}}\right)^{-1} \mathbf{B}_{\mathrm{c}}^{\mathrm{T}} \mathbf{W}_{\mathrm{c}} \mathbf{d} .
\end{aligned}
$$


Validation of (37) and (38) are covered in Section 5.1 and 5.3 respectively.

If weightings are chosen optimally as $\mathbf{U}_{\mathrm{s}}=\mathbf{0}, \mathbf{U}_{\mathrm{c}}=\mathbf{0}, \mathbf{W}_{\mathrm{s}}=\left(\operatorname{cov}\left(\boldsymbol{\omega}^{2}\right)\right)^{-1}$ and $\mathbf{W}_{\mathrm{c}}=(\operatorname{cov}(\mathbf{d}))^{-1}$

[16], the covariances of estimates are [16]

$$
\operatorname{cov}\left(\hat{\mathbf{p}}_{\mathrm{s}}\right)=\left(\mathbf{B}_{\mathrm{s}}^{\mathrm{T}}\left(\operatorname{cov}\left(\boldsymbol{\omega}^{2}\right)\right)^{-1} \mathbf{B}_{\mathrm{s}}\right)^{-1}, \operatorname{cov}\left(\hat{\mathbf{p}}_{\mathrm{c}}\right)=\left(\mathbf{B}_{\mathrm{c}}^{\mathrm{T}}(\operatorname{cov}(\mathbf{d}))^{-1} \mathbf{B}_{\mathrm{c}}\right)^{-1},
$$

which however excludes variability of $\mathbf{B}_{\mathrm{s}}, \mathbf{B}_{\mathrm{c}}$ and $\boldsymbol{\omega}_{0}^{2}$ due to uncertainty of measured mode shapes. Equations (39) show how measurement noise in $\boldsymbol{\omega}^{2}$ and $\mathbf{d}$ result in estimation uncertainty in $\hat{\mathbf{p}}_{\mathrm{s}}$ and $\hat{\mathbf{p}}_{\mathrm{c}}$. Estimation uncertainty is large when matrices to be inverted in (39) are close to singular, empirically found to occur when two beam boundaries are identical, or when mode shapes or their slopes are zero at boundaries due to very large boundary stiffness, or when stiffness parameters or tension are very small.

When unable to estimate parameters accurately due to singularity of the matrices inverted in (37)-(38), nonzero $\mathbf{U}_{\mathrm{s}}$ and $\mathbf{U}_{\mathrm{c}}$ should be used. For example if damping parameters cannot be estimated due to nearly identical boundaries, $\mathbf{U}_{\mathrm{c}}$ can be chosen so the quadratic in $\sqrt{36},, \hat{\mathbf{p}}_{\mathrm{c}}^{\mathrm{T}} \mathbf{U}_{\mathrm{c}} \hat{\mathbf{p}}_{\mathrm{c}}$, equals e.g. $10^{6}\left(\left(\hat{c}_{1}-\hat{c}_{2}\right)^{2}+\left(\hat{c}_{3}-\hat{c}_{4}\right)^{2}\right)$; then minimization of $S_{\mathrm{c}}$ will tend to give $\hat{c}_{1} \approx \hat{c}_{2}$ and $\hat{c}_{3} \approx \hat{c}_{4}$

\subsection{Summary}

The suggested Method 1 estimates tension, boundary stiffness, and boundary damping by fitting natural frequency, damping coefficient and mode shape data to a theoretical relationship derived from Rayleigh's quotient. Estimation accuracy is low when boundary stiffness are very large or very small, when tension is very small, or when boundaries are identical.

Method 1 requires measurements of continuous mode shapes, which are typically not measurable, i.e. must be fitted using discrete mode shapes or otherwise estimated. The next section suggests a method to estimate tension and boundary stiffness without continuous mode shapes, through nonlinear regression.

\section{Method 2: Boundary stiffness parameter estimation using nonlinear regression}

Here we suggest a method for estimating only tension and boundary stiffness, using nonlinear regression to fit parameters to measured natural frequencies and/or mode shapes, through the eigenvalue problem solution (28) and (20)-(22). This method assumes small damping and does not require knowledge of mode shapes. An object function to be minimized is defined:

$$
\begin{array}{r}
S\left(\hat{\mathbf{p}}_{\mathrm{s}}\right)=\left(\boldsymbol{\omega}-\hat{\boldsymbol{\omega}}\left(\hat{\mathbf{p}}_{\mathrm{s}}\right)\right)^{\mathrm{T}} \mathbf{W}\left(\boldsymbol{\omega}-\hat{\boldsymbol{\omega}}\left(\hat{\mathbf{p}}_{\mathrm{s}}\right)\right)+\left(\tilde{\mathbf{p}}_{\mathrm{s}}-\hat{\mathbf{p}}_{\mathrm{s}}\right)^{\mathrm{T}} \mathbf{U}\left(\tilde{\mathbf{p}}_{\mathrm{s}}-\hat{\mathbf{p}}_{\mathrm{s}}\right) \\
+\gamma \sum_{j=1}^{n} \int_{0}^{1} w_{j}(x)\left(\phi_{j}(x)-\hat{\phi}_{j}\left(x, \hat{\mathbf{p}}_{\mathrm{s}}\right)\right)^{2} d x .
\end{array}
$$

Here the first term represents deviations between $n$ natural frequencies measured, $\boldsymbol{\omega} \in \mathbb{R}^{n}$, and predicted, $\hat{\boldsymbol{\omega}} \in \mathbb{R}^{n}$, from a parameter estimate $\hat{\mathbf{p}}_{\mathrm{s}}=\left\{\begin{array}{lllll}p & k_{1} & k_{2} & k_{3} & k_{4}\end{array}\right\}^{\mathrm{T}}$. The third term represents the same, but for mode shapes measured, $\phi_{j}(x) \in \mathbb{R}$, and predicted $\hat{\phi}_{j}(x) \in \mathbb{R}$ for $n$ modes. 
The second term represents deviation between an a posteriori estimate of parameters $\tilde{\mathbf{p}}_{\mathrm{s}}$ and a new

estimate $\hat{\mathbf{p}}_{\mathrm{s}}$. The a posteriori estimate $\tilde{\mathbf{p}}_{\mathrm{s}}$ is based on previous experiences. Weightings $\mathbf{W} \in \mathbb{R}^{n \times n}$, $\mathbf{U} \in \mathbb{R}^{5 \times 5}$ and $w_{j}(x) \in \mathbb{R}$ represent confidence in each type of data. The variable $\gamma$ weighs mode shape deviations relative to other deviations, cf. Section 4.1. Mode shapes can typically only be measured at discrete locations, $x_{i}$, in which case $w_{j}(x)$ is a sum of Dirac delta functions:

$$
w_{j}(x)=\sum_{i} w_{i j} \delta\left(x-x_{i}\right)
$$

Optimally, the weights should be chosen as inverse covariances of measurements [16], i.e. $\mathbf{W}=(\operatorname{cov}(\boldsymbol{\omega}))^{-1}, \mathbf{U}=\left(\operatorname{cov}\left(\tilde{\mathbf{p}}_{\mathrm{s}}\right)\right)^{-1}$, and $w_{i j}=\left(\operatorname{var}\left(\phi_{j}\left(x_{i}\right)\right)\right)^{-1}$; if mode shapes are not measured $w_{j}(x)=0$.

The object function $S$ will be minimized by setting $\partial S / \partial \mathbf{p}_{\mathrm{s}}=\mathbf{0}$, and using Gauss-Newton iteration [16] to solve for $\hat{\mathbf{p}}_{\mathrm{s}}$. The gradient $\partial S / \partial \mathbf{p}_{\mathrm{s}}$ is linearized around an estimate $\hat{\mathbf{p}}_{\mathrm{s}, k}$ after $k$ iterations:

$$
\frac{\partial S\left(\hat{\mathbf{p}}_{\mathrm{s}}\right)}{\partial \mathbf{p}_{\mathrm{s}}}=-2 \mathbf{h}\left(\hat{\mathbf{p}}_{\mathrm{s}, k}\right)-2 \mathbf{H}\left(\hat{\mathbf{p}}_{\mathrm{s}, k}\right)\left(\hat{\mathbf{p}}_{\mathrm{s}}-\hat{\mathbf{p}}_{\mathrm{s}, k}\right)+O\left(\left(\hat{\mathbf{p}}_{\mathrm{s}}-\hat{\mathbf{p}}_{\mathrm{s}, k}\right)^{\mathrm{T}}\left(\hat{\mathbf{p}}_{\mathrm{s}}-\hat{\mathbf{p}}_{\mathrm{s}, k}\right)\right),
$$

which is solved for a new estimate $\hat{\mathbf{p}}_{\mathrm{s}}=\hat{\mathbf{p}}_{\mathrm{s}, k+1}$ from the condition $\partial S / \partial \mathbf{p}_{\mathrm{s}}=\mathbf{0}$ [16]:

$$
\hat{\mathbf{p}}_{\mathrm{s}, k+1}=\hat{\mathbf{p}}_{\mathrm{s}, k}+h_{k} \mathbf{H}\left(\hat{\mathbf{p}}_{\mathrm{s}, k}\right)^{-1} \mathbf{h}\left(\hat{\mathbf{p}}_{\mathrm{s}, k}\right)
$$

where $\mathbf{H} \in \mathbb{R}^{5 \times 5}$ and $\mathbf{h} \in \mathbb{R}^{5}$,

$$
\mathbf{H}\left(\hat{\mathbf{p}}_{\mathrm{s}, k}\right)=\frac{\partial \hat{\boldsymbol{\omega}}\left(\hat{\mathbf{p}}_{\mathrm{s}, k}\right)^{\mathrm{T}}}{\partial \hat{\mathbf{p}}_{\mathrm{s}}} \mathbf{W} \frac{\partial \hat{\boldsymbol{\omega}}\left(\hat{\mathbf{p}}_{\mathrm{s}, k}\right)}{\partial \hat{\mathbf{p}}_{\mathrm{s}}}+\mathbf{U}+\gamma \sum_{j=1}^{n} \int_{0}^{1} w_{j}(x) \frac{\partial \phi_{j}\left(x, \hat{\mathbf{p}}_{\mathrm{s}, k}\right)^{\mathrm{T}}}{\partial \hat{\mathbf{p}}_{\mathrm{s}}} \frac{\partial \phi_{j}\left(x, \hat{\mathbf{p}}_{\mathrm{s}, k}\right)}{\partial \hat{\mathbf{p}}_{\mathrm{s}}} d x
$$

and

$$
\begin{aligned}
& \mathbf{h}\left(\hat{\mathbf{p}}_{\mathrm{s}, k}\right)=\frac{\partial \hat{\boldsymbol{\omega}}\left(\hat{\mathbf{p}}_{\mathrm{s}, k}\right)^{\mathrm{T}}}{\partial \hat{\mathbf{p}}_{\mathrm{s}}} \mathbf{W}\left(\boldsymbol{\omega}-\hat{\boldsymbol{\omega}}\left(\hat{\mathbf{p}}_{\mathrm{s}, k}\right)\right)+\mathbf{U}\left(\tilde{\mathbf{p}}_{\mathrm{s}}-\hat{\mathbf{p}}_{\mathrm{s}, k}\right) \\
& +\gamma \sum_{j=1}^{n} \int_{0}^{1} w_{j}(x) \frac{\partial \hat{\phi}_{j}\left(x, \hat{\mathbf{p}}_{\mathrm{s}, k}\right)^{\mathrm{T}}}{\partial \hat{\mathbf{p}}_{\mathrm{s}}}\left(\phi_{j}(x)-\hat{\phi}_{j}\left(x, \hat{\mathbf{p}}_{\mathrm{s}, k}\right)\right) d x,
\end{aligned}
$$

are related to the second and first derivatives of $S$ w.r.t. parameters $\hat{\mathbf{p}}$. The scalar $h_{k}$ in (43) takes the quadratic (last) term in (42) into account via Box-Kanemasu's method [16], which chooses

$$
h_{k}=\left\{\begin{array}{cc}
1 & 2 a_{2}+a_{1} \leq 0 \\
-\frac{a_{1}}{2 a_{2}} & 2 a_{2}+a_{1}>0
\end{array},\right.
$$

where

$$
a_{1}=-2 \mathbf{h}\left(\hat{\mathbf{p}}_{\mathrm{s}, k}\right)^{\mathrm{T}} \mathbf{H}\left(\hat{\mathbf{p}}_{\mathrm{s}, k}\right)^{-1} \mathbf{h}\left(\hat{\mathbf{p}}_{\mathrm{s}, k}\right), \quad a_{2}=S\left(\hat{\mathbf{p}}_{\mathrm{s}, k}+\mathbf{H}\left(\hat{\mathbf{p}}_{\mathrm{s}, k}\right)^{-1} \mathbf{h}\left(\hat{\mathbf{p}}_{\mathrm{s}, k}\right)\right)-S\left(\hat{\mathbf{p}}_{\mathrm{s}, k}\right)-a_{1} .
$$

Repeated use of (43) produces gradually improved estimates of $\mathbf{p}_{\mathrm{s}}$. The calculation of components $\mathbf{H}, \mathbf{h}$ and $h_{k}$ are given below, cf. Section 4.2. 


\subsection{Local minimum near reversed boundary}

A problem arises when estimating $\hat{\mathbf{p}}_{\mathrm{s}}$ by iterating (43). If true parameters are $p$ and $k_{1-4}$, a local minimum for $S\left(\hat{\mathbf{p}}_{\mathrm{s}}\right)$ appears at $\hat{p}=p, \hat{k}_{1}=k_{2}, \hat{k}_{2}=k_{1}, \hat{k}_{3}=k_{4}, \hat{k}_{4}=k_{3}$ due to natural frequencies not distinguishing left and right boundaries. Mode shapes, if known, can distinguish boundaries, so to avoid this local minimum, one can use a large multiplier $\gamma$ in (40) to weigh deviations in mode shapes high for initial iterations with (43), after which $\gamma$ is unity. We suggest

$$
\gamma=1+\frac{\gamma_{\max }-1}{2^{k}}
$$

which has been found to work well for cases studied, for $\gamma_{\max }=100$. Variable $k$ is the number of iterations passed, so $\gamma$ is initially $\gamma_{\max }$ and converges to 1 for increasing $k$.

\subsection{Computing corrected parameters}

To use (43), scripts must be created, for performing these steps:

1) Calculate natural frequencies $\hat{\omega}_{j}$ and boundary condition matrix $\mathbf{S}_{j}$ for modes $j=1,2, \ldots n$ by solving (28) and inserting into (23).

2) Calculate unscaled mode shape coefficients $\overline{\mathbf{q}}_{j}$ for each mode as the unit eigenvector of $\mathbf{S}_{j}$ from (23).

3) Scale mode shape coefficients by a scalar $s_{j}$ for each mode, $\mathbf{q}_{j}=s_{j} \overline{\mathbf{q}}_{j}$, so predicted and measured mode shapes $\hat{\phi}_{j}\left(x, \hat{\mathbf{p}}_{\mathrm{s}}\right)$ and $\phi_{j}(x)$ are of similar magnitude.

4) Calculate derivatives $\partial \hat{\boldsymbol{\omega}}_{j}\left(\hat{\mathbf{p}}_{\mathrm{s}}\right) / \partial \hat{\mathbf{p}}_{\mathrm{s}}$ and $\partial \hat{\phi}_{j}\left(x, \hat{\mathbf{p}}_{\mathrm{s}}\right) / \partial \hat{\mathbf{p}}_{\mathrm{s}}$.

5) Calculate $S$.

6) Calculate $\mathbf{h}, \mathbf{H}$ and $h_{k}$

7) Load measured natural frequencies and/or mode shapes, define $\mathbf{W}, \mathbf{U}, w_{j}(x), \tilde{\mathbf{p}}_{\mathrm{s}}$, initial value $\hat{\mathbf{p}}_{\mathrm{s}, 0}$, and repeatedly use (43) until convergence.

\subsubsection{Re. Step 3, scaling mode shapes}

Estimated mode shape coefficients $\mathbf{q}_{j}=s_{j} \overline{\mathbf{q}}_{j}$ are scaled with $s_{j}$ to make estimated mode shapes

$$
\hat{\phi}_{j}\left(x, \hat{\mathbf{p}}_{\mathrm{s}}\right)=s_{j} \lambda\left(x, \hat{p}, \hat{\omega}_{j}\right)^{\mathrm{T}} \overline{\mathbf{q}}_{j}
$$

of similar magnitude as measured mode shapes $\phi_{j}(x)$, by minimizing a new object function,

$$
S_{\phi, j}\left(s_{j}\right)=\int_{0}^{1} w_{j}(x)\left(s_{j} \lambda\left(x, \hat{p}, \hat{\omega}_{j}\right)^{\mathrm{T}} \overline{\mathbf{q}}_{j}-\phi_{j}(x)\right)^{2} d x,
$$

which describes the deviation between predicted and measured mode shape. Minimizing (50) gives

$$
s_{j}=\frac{\int_{0}^{1} w_{j}(x) \phi_{j}(x) \lambda\left(x, \hat{p}, \hat{\omega}_{j}\right)^{\mathrm{T}} \overline{\mathbf{q}}_{j} d x}{\int_{0}^{1} w_{j}(x)\left(\lambda\left(x, \hat{p}, \hat{\omega}_{j}\right)^{\mathrm{T}} \overline{\mathbf{q}}_{j}\right)^{2} d x}=\frac{\mathbf{g}_{j}\left[\phi_{j}(x)\right]^{\mathrm{T}} \overline{\mathbf{q}}_{j}}{\overline{\mathbf{q}}_{j}^{\mathrm{T}} \mathbf{G}_{j}[1] \overline{\mathbf{q}}_{j}}
$$


where $\mathbf{g}_{j}[f(x)] \in \mathbb{R}^{4}$ and $\mathbf{G}_{j}[f(x)] \in \mathbb{R}^{4 \times 4}$ are functionals, integrals converted to summations through manipulations of the Dirac delta function in (41), defined as

$$
\begin{aligned}
\mathbf{g}_{j}[f(x)] & =\sum_{i} w_{i j} f\left(x_{i}\right) \lambda\left(x, \hat{p}, \hat{\omega}_{j}\right), \\
\mathbf{G}_{j}[f(x)] & =\sum_{i} w_{i j} f\left(x_{i}\right) \lambda\left(x_{i}, \hat{p}, \hat{\omega}_{j}\right) \lambda\left(x_{i}, \hat{p}, \hat{\omega}_{j}\right)^{\mathrm{T}} .
\end{aligned}
$$

Functionals $\mathbf{G}$ and $\mathbf{g}$ are scripted as subroutines, as these types of summations reappear in other calculations.

4.2.2. Re. Step 4, partial derivative of natural frequencies

Differentiating (28) w.r.t. parameters $\hat{\mathbf{p}}_{\mathrm{s}}$ and solving for $\partial \hat{\omega}_{j}\left(\hat{\mathbf{p}}_{\mathrm{s}}\right) / \partial \hat{\mathbf{p}}_{\mathrm{s}}$, gives

$$
\frac{\partial \hat{\omega}_{j}\left(\hat{\mathbf{p}}_{\mathrm{s}}\right)}{\partial \hat{\mathbf{p}}_{\mathrm{s}}}=-\frac{\mathbf{r}^{\mathrm{T}} \frac{\partial \mathbf{k}}{\partial \hat{\mathbf{p}}_{\mathrm{s}}}+\mathbf{k}^{\mathrm{T}} \frac{\partial \mathbf{r}}{\partial \hat{p}} \frac{\partial \hat{p}}{\partial \hat{\mathbf{p}}_{\mathrm{s}}}}{\mathbf{k}^{\mathrm{T}} \frac{\partial \mathbf{r}}{\partial \hat{\omega}_{j}}},
$$

where $\partial \mathbf{k} / \partial \hat{\mathbf{p}}_{\mathrm{s}}$ is calculated by differentiating $(26), \partial \hat{p} / \partial \hat{\mathbf{p}}_{\mathrm{s}}=\left\{\begin{array}{lllll}1 & 0 & 0 & 0 & 0\end{array}\right\}$ and the derivatives of $\mathbf{r}$ are calculated as

$$
\frac{\partial \mathbf{r}}{\partial \hat{\omega}_{j}}=\frac{\partial \mathbf{r}}{\partial \alpha_{1, j}} \frac{\partial \alpha_{1, j}}{\partial \hat{\omega}_{j}}+\frac{\partial \mathbf{r}}{\partial \alpha_{2, j}} \frac{\partial \alpha_{2, j}}{\partial \hat{\omega}_{j}}, \quad \frac{\partial \mathbf{r}}{\partial \hat{p}}=\frac{\partial \mathbf{r}}{\partial \alpha_{1, j}} \frac{\partial \alpha_{1, j}}{\partial \hat{p}}+\frac{\partial \mathbf{r}}{\partial \alpha_{2, j}} \frac{\partial \alpha_{2, j}}{\partial \hat{p}},
$$

where derivatives of $\mathbf{r}$ w.r.t. $\alpha_{1, j}$ and $\alpha_{2, j}$ are calculated numerically from (29), and derivatives of $\alpha_{1, j}$ and $\alpha_{2, j}$ are calculated analytically from differentiating (21). The derivative of $\hat{\boldsymbol{\omega}}$ is then

$$
\frac{\partial \hat{\boldsymbol{\omega}}\left(\hat{\mathbf{p}}_{\mathrm{s}}\right)}{\partial \hat{\mathbf{p}}_{\mathrm{s}}}=\left\{\begin{array}{c}
\partial \hat{\omega}_{1}\left(\hat{\mathbf{p}}_{\mathrm{s}}\right) / \partial \hat{\mathbf{p}}_{\mathrm{s}} \\
\ldots \\
\partial \hat{\omega}_{n}\left(\hat{\mathbf{p}}_{\mathrm{s}}\right) / \partial \hat{\mathbf{p}}_{\mathrm{s}}
\end{array}\right\} .
$$

4.2.3. Re. Step 4, partial derivative of mode shapes

The derivative $\partial \hat{\phi}_{j}\left(x, \hat{\mathbf{p}}_{\mathrm{s}}\right) / \partial \hat{\mathbf{p}}_{\mathrm{s}}$ by differentiation of $[49]$, is

$$
\frac{\partial \hat{\phi}_{j}\left(x, \hat{\mathbf{p}}_{\mathrm{s}}\right)}{\partial \hat{\mathbf{p}}_{\mathrm{s}}}=\frac{\partial s_{j}}{\partial \hat{\mathbf{p}}_{\mathrm{s}}} \lambda\left(x, \hat{p}, \hat{\omega}_{j}\right)^{\mathrm{T}} \overline{\mathbf{q}}_{j}+s_{j} \frac{\partial \lambda^{\mathrm{T}}}{\partial \hat{\mathbf{p}}_{\mathrm{s}}} \overline{\mathbf{q}}_{j}+s_{j} \lambda\left(x, \hat{p}, \hat{\omega}_{j}\right)^{\mathrm{T}} \frac{\partial \overline{\mathbf{q}}_{j}}{\partial \hat{\mathbf{p}}_{\mathrm{s}}} .
$$

Differentials $\mathrm{d} s_{j}, \mathrm{~d} \lambda$ and d $\overline{\mathbf{q}}_{j}$ will be derived for use in (57). Taking the differential of $\lambda\left(x, \hat{p}, \hat{\omega}_{j}\right)=$ $\left\{\begin{array}{llll}\cos \left(\alpha_{1, j} x\right) & \sin \left(\alpha_{1, j} x\right) & \cosh \left(\alpha_{2, j} x\right) & \sinh \left(\alpha_{2, j} x\right)\end{array}\right\}^{\mathrm{T}}$ gives

$$
\mathrm{d} \lambda=\mathrm{d} \alpha \lambda\left(x, \hat{p}, \hat{\omega}_{j}\right) x
$$

where $\alpha \in \mathbb{R}^{4 \times 4}$ is

$$
\boldsymbol{\alpha}=\left[\begin{array}{cccc}
0 & -\alpha_{1, j} & 0 & 0 \\
\alpha_{1, j} & 0 & 0 & 0 \\
0 & 0 & 0 & \alpha_{2, j} \\
0 & 0 & \alpha_{2, j} & 0
\end{array}\right]
$$


Taking the differentials of (52)-(53) and inserting (58) gives $\mathrm{d} \mathbf{g}[f(x)]=\mathrm{d} \boldsymbol{\alpha} \mathbf{g}[x f(x)]$ and $\mathrm{d} \mathbf{G}[f(x)]=\mathrm{d} \boldsymbol{\alpha} \mathbf{G}[x f(x)]+\mathbf{G}[x f(x)] \mathrm{d} \boldsymbol{\alpha}^{\mathrm{T}}$, which are used for taking the differential of (51), which gives:

$$
\mathrm{d} s_{j}=\mathbf{z}_{1 j}^{\mathrm{T}} \mathrm{d} \overline{\mathbf{q}}_{j}+\mathbf{z}_{2 j}^{\mathrm{T}} \mathrm{d} \alpha \overline{\mathbf{q}}_{j}
$$

where $\mathbf{z}_{1 j}, \mathbf{z}_{2 j} \in \mathbb{R}^{4}$ are

$$
\mathbf{z}_{1 j}=\frac{\mathbf{g}_{j}\left[\phi_{j}(x)\right]-2 s_{j} \mathbf{G}_{j}[1] \overline{\mathbf{q}}_{j}}{\left(\overline{\mathbf{q}}_{j}^{\mathrm{T}} \mathbf{G}_{j}[1] \overline{\mathbf{q}}_{j}\right)^{2}}, \quad \mathbf{z}_{2 j}=\frac{\mathbf{g}_{j}\left[x \phi_{j}(x)\right]-2 s_{j} \mathbf{G}_{j}[x] \overline{\mathbf{q}}_{j}}{\left(\overline{\mathbf{q}}_{j}^{\mathrm{T}} \mathbf{G}_{j}[1] \overline{\mathbf{q}}_{j}\right)^{2}} .
$$

Only $\overline{\mathbf{q}}_{j}$ remains to be an explicit function of parameters to evaluate $(57)$, but is only implicitly defined to be the unit eigenvector satisfying $(22), \mathbf{S}_{j} \overline{\mathbf{q}}_{j}=\mathbf{0}$, and $\overline{\mathbf{q}}_{j}^{\mathrm{T}} \overline{\mathbf{q}}_{j}=1$. Taking the differential of these conditions gives

$$
\mathrm{d} \mathbf{S}_{j} \overline{\mathbf{q}}_{j}+\mathbf{S}_{j} \mathrm{~d} \overline{\mathbf{q}}_{j}=\mathbf{0}, \quad \mathrm{d} \overline{\mathbf{q}}_{j}^{\mathrm{T}} \overline{\mathbf{q}}_{j}=0,
$$

which has only a single solution $\mathrm{d} \overline{\mathbf{q}}_{j}=-\mathbf{S}_{j}^{\mathrm{T}}\left(\mathbf{S}_{j} \mathbf{S}_{j}^{\mathrm{T}}\right)^{-1} \mathrm{~d} \mathbf{S}_{j} \overline{\mathbf{q}}_{j}$, so that

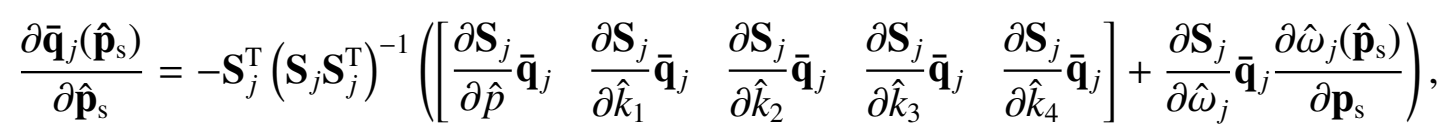

where derivatives of $\mathbf{S}_{j}=\mathbf{S}_{j}\left(\hat{\mathbf{p}}_{\mathrm{s}}, \hat{\omega}_{j}\right)$ w.r.t. parameters have both an explicit part and an implicit part due to $\mathbf{S}_{j}$ 's dependence on $\hat{\omega}_{j}\left(\hat{\mathbf{p}}_{\mathbf{s}}\right)$. The derivatives $\partial \mathbf{S}_{j} / \partial \hat{k}_{1-4}$ are calculated by analytic differentiation of (23). The derivatives $\partial \mathbf{S}_{j} / \partial \hat{p}$ and $\partial \mathbf{S}_{j} / \partial \hat{\omega}_{j}$ are calculated as

$$
\frac{\partial \mathbf{S}_{j}}{\partial \hat{\omega}_{j}}=\frac{\partial \mathbf{S}_{j}}{\partial \alpha_{1, j}} \frac{\partial \alpha_{1, j}}{\partial \hat{\omega}_{j}}+\frac{\partial \mathbf{S}_{j}}{\partial \alpha_{2, j}} \frac{\partial \alpha_{2, j}}{\partial \hat{\omega}_{j}}, \quad \frac{\partial \mathbf{S}_{j}}{\partial \hat{p}}=\frac{\partial \mathbf{S}_{j}}{\partial \alpha_{1, j}} \frac{\partial \alpha_{1, j}}{\partial \hat{p}}+\frac{\partial \mathbf{S}_{j}}{\partial \alpha_{2, j}} \frac{\partial \alpha_{2, j}}{\partial \hat{p}},
$$

where derivatives of $\mathbf{S}_{j}$ w.r.t. $\alpha_{1, j}$ and $\alpha_{2, j}$ are found by differentiating $(23)$ numerically, and derivatives of $\alpha_{1, j}$ and $\alpha_{2, j}$ are found analytically by differentiating (21).

Inserting (58) and (60) into (57) gives

$$
\frac{\partial \hat{\phi}_{j}\left(x, \hat{\mathbf{p}}_{\mathrm{s}}\right)}{\partial \hat{\mathbf{p}}_{\mathrm{s}}}=\lambda\left(x, \hat{p}, \hat{\omega}_{j}\right)^{\mathrm{T}}\left(\mathbf{Z}_{1 j}+\mathbf{Z}_{2 j} x\right)
$$

where $\mathbf{Z}_{1 j}, \mathbf{Z}_{2 j} \in \mathbb{R}^{4 \times 5}$ are matrices:

$$
\begin{aligned}
& \mathbf{Z}_{1 j}=\overline{\mathbf{q}}_{j} \mathbf{z}_{2 j}^{\mathrm{T}}\left(\frac{\partial \boldsymbol{\alpha}^{\mathrm{T}}}{\partial \hat{p}} \overline{\mathbf{q}}_{j} \frac{\partial \hat{p}}{\partial \hat{\mathbf{p}}_{\mathrm{s}}}+\frac{\partial \boldsymbol{\alpha}^{\mathrm{T}}}{\partial \hat{\omega}_{j}} \overline{\mathbf{q}}_{j} \frac{\partial \hat{\omega}_{j}\left(\hat{\mathbf{p}}_{\mathrm{s}}\right)}{\partial \hat{\mathbf{p}}_{\mathrm{s}}}\right)+\overline{\mathbf{q}}_{j} \mathbf{z}_{1 j}^{\mathrm{T}} \frac{\partial \overline{\mathbf{q}}_{j}}{\partial \hat{\mathbf{p}}_{\mathrm{s}}}+s_{j} \frac{\partial \overline{\mathbf{q}}_{j}}{\partial \hat{\mathbf{p}}_{\mathrm{s}}}, \\
& \mathbf{Z}_{2 j}=s_{j}\left(\frac{\partial \boldsymbol{\alpha}^{\mathrm{T}}}{\partial \hat{p}} \overline{\mathbf{q}}_{j} \frac{\partial \hat{p}}{\partial \hat{\mathbf{p}}_{\mathrm{s}}}+\frac{\partial \boldsymbol{\alpha}^{\mathrm{T}}}{\partial \hat{\omega}_{j}} \overline{\mathbf{q}}_{j} \frac{\partial \hat{\omega}_{j}}{\partial \hat{\mathbf{p}}_{\mathrm{s}}}\right) .
\end{aligned}
$$

4.2.4. Re. Step 5, evaluating the object function

Inserting (41) and (49) into (40) gives an expression for $S$ suitable for computation:

$$
\begin{aligned}
S\left(\hat{\mathbf{p}}_{\mathrm{s}}\right)= & \left(\boldsymbol{\omega}-\hat{\mathbf{\omega}}\left(\hat{\mathbf{p}}_{\mathrm{s}}\right)\right)^{\mathrm{T}} \mathbf{W}\left(\boldsymbol{\omega}-\hat{\mathbf{\omega}}\left(\hat{\mathbf{p}}_{\mathrm{s}}\right)\right)+\left(\tilde{\mathbf{p}}_{\mathrm{s}}-\hat{\mathbf{p}}_{\mathrm{s}}\right)^{\mathrm{T}} \mathbf{U}\left(\tilde{\mathbf{p}}_{\mathrm{s}}-\hat{\mathbf{p}}_{\mathrm{s}}\right) \\
& +\gamma \sum_{j=1}^{n} \sum_{i} w_{i j}\left(\phi_{j}\left(x_{i}\right)-s_{j} \lambda\left(x_{i}, \hat{p}, \hat{\omega}_{j}\right)^{\mathrm{T}} \overline{\mathbf{q}}_{j}\right)^{2} .
\end{aligned}
$$




\subsubsection{Re. Step 6, evaluating $\mathbf{H}$ and $\mathbf{h}$}

To calculate $\mathbf{h}$ and $\mathbf{H}$, insertion of (65) into (44)-(45), and using defined functionals (52)-(53) gives

$$
\begin{aligned}
\mathbf{H}\left(\hat{\mathbf{p}}_{\mathrm{s}, k}\right)= & \frac{\partial \hat{\mathbf{w}}\left(\hat{\mathbf{p}}_{\mathrm{s}, k}\right)^{\mathrm{T}}}{\partial \hat{\mathbf{p}}_{\mathrm{s}}} \mathbf{W} \frac{\partial \hat{\mathbf{\omega}}\left(\hat{\mathbf{p}}_{\mathrm{s}, k}\right)}{\partial \hat{\mathbf{p}}_{\mathrm{s}}}+\mathbf{U}+ \\
& \gamma \sum_{j=1}^{n}\left(\mathbf{Z}_{1 j}^{\mathrm{T}} \mathbf{G}_{j}[1] \mathbf{Z}_{1 j}+\mathbf{Z}_{1 j}^{\mathrm{T}} \mathbf{G}_{j}[x] \mathbf{Z}_{2 j}+\mathbf{Z}_{2 j}^{\mathrm{T}} \mathbf{G}_{j}[x] \mathbf{Z}_{1 j}+\mathbf{Z}_{2 j}^{\mathrm{T}} \mathbf{G}_{j}\left[x^{2}\right] \mathbf{Z}_{2 j}\right)
\end{aligned}
$$

and

$$
\begin{aligned}
\mathbf{h}\left(\hat{\mathbf{p}}_{\mathrm{s}, k}\right)= & \frac{\partial \hat{\mathbf{\omega}}\left(\hat{\mathbf{p}}_{\mathrm{s}, k}\right)^{\mathrm{T}}}{\partial \hat{\mathbf{p}}_{\mathrm{s}}} \mathbf{W}\left(\boldsymbol{\omega}-\hat{\boldsymbol{\omega}}\left(\hat{\mathbf{p}}_{\mathrm{s}, k}\right)\right)+\mathbf{U}\left(\tilde{\mathbf{p}}_{\mathrm{s}}-\hat{\mathbf{p}}_{\mathrm{s}, k}\right)+ \\
& \gamma \sum_{j=1}^{n}\left(\mathbf{Z}_{1 j}^{\mathrm{T}}\left(\mathbf{g}_{j}\left[\phi_{j}(x)\right]-s_{j}\left(\hat{\mathbf{p}}_{\mathrm{s}}\right) \mathbf{G}_{j}[1] \overline{\mathbf{q}}_{j}\left(\hat{\mathbf{p}}_{\mathrm{s}}\right)\right)+\mathbf{Z}_{2 j}^{\mathrm{T}}\left(\mathbf{g}_{j}\left[x \phi_{j}(x)\right]-s_{j}\left(\hat{\mathbf{p}}_{\mathrm{s}}\right) \mathbf{G}_{j}[x] \overline{\mathbf{q}}_{j}\left(\hat{\mathbf{p}}_{\mathrm{s}}\right)\right)\right) .
\end{aligned}
$$

Equations (69)-(70) are more convenient than (44)-(45), as integrations are compressed into functionals $\mathbf{g}$ and $\mathbf{G}$ of scalar functions, which can be scripted as subroutines.

\subsubsection{Re. Step 7, iteration stability and convergence}

What remains is a script that loads measurements and chooses $\mathbf{W}, w_{i j}, \mathbf{U}, \tilde{\mathbf{p}}_{\mathrm{s}}$, and initial parameters $\hat{\mathbf{p}}_{\mathrm{s}, 0}$. Initial parameters $\hat{\mathbf{p}}_{\mathrm{s}, 0}$ should be chosen neither small or large, because this would slow iterations by making $\mathbf{h} \approx \mathbf{0}$.

Without a nonzero a posteriori confidence $\mathbf{U}$ in $(69)-(70)$, the iteration scheme $(43)$ diverged for the cases studied. Marquardt and Levenberg suggest choosing a posteriori estimate $\tilde{\mathbf{p}}_{\mathrm{s}}=$ $\hat{\mathbf{p}}_{\mathrm{s}, k}$, so there is confidence in previous iteration of parameters, dampening unstable iterations. Consequently, $\mathbf{U}$ has no effect on $\mathbf{h}$ in (70). Also, Marquardt and Levenberg suggest choosing [16]

$$
\mathbf{U}=\lambda_{k} \boldsymbol{\Omega}\left(\hat{\mathbf{p}}_{\mathrm{s}, k}\right),
$$

where $\lambda_{k}$ is a relaxation parameter describing relative confidence between $\tilde{\mathbf{p}}_{\mathrm{s}}$ and measurements, while $\boldsymbol{\Omega}$ is a diagonal matrix consisting of the diagonal elements of $\mathbf{H}-\mathbf{U}$ from (69), so the magnitudes of U's elements are comparable to the rest of the components of $\mathbf{H}$. Levenberg and Marquardt suggest different relaxation parameters, $\lambda_{1}$, and $\lambda_{\mathrm{m}}$ :

$$
\lambda_{1}=\frac{\mathbf{h}\left(\hat{\mathbf{p}}_{\mathrm{s}, k}\right)^{\mathrm{T}} \mathbf{\Omega}\left(\hat{\mathbf{p}}_{\mathrm{s}, k}\right) \mathbf{h}\left(\hat{\mathbf{p}}_{\mathrm{s}, k}\right)}{S\left(\hat{\mathbf{p}}_{\mathrm{s}, k}\right)}, \lambda_{\mathrm{m}}=\frac{\lambda_{0}}{v^{k}} .
$$

Levenberg's choice reduces step sizes in (43) proportional to $\mathbf{h}$ and converges to zero near local minimums $\mathbf{h} \approx \mathbf{0}$ for $S$ [16]. Marquardt's choice initially gives large confidence in $\tilde{\mathbf{p}}_{\mathrm{s}}$, reducing step sizes of iterations, then relaxes $\lambda_{k}$ so confidence in $\tilde{\mathbf{p}}_{\mathrm{s}}$ converges to zero.

From numerical experiments using (43), we suggest

$$
\lambda_{k}=\left\{\begin{array}{cl}
\lambda_{1} \lambda_{\mathrm{m}} & S\left(\hat{\mathbf{p}}_{\mathrm{s}, k}\right) \geq \kappa E\left[S\left(\mathbf{p}_{\mathrm{s}}\right)\right] \\
\lambda_{1} & S\left(\hat{\mathbf{p}}_{\mathrm{s}, k}\right)<\kappa E\left[S\left(\mathbf{p}_{\mathrm{s}}\right)\right]
\end{array},\right.
$$


with $\lambda_{0}=100, v=10$ in $(72)$, where the expectation $E\left[S\left(\mathbf{p}_{\mathrm{s}}\right)\right]$ is an estimate of what $S$ is for true parameters $\mathbf{p}_{\mathrm{s}}$, and $\kappa$, here suggested as $\kappa=3 / 2$, is a number taking into account $S\left(\hat{\mathbf{p}}_{\mathrm{s}}\right)$ not being minimal at its expected value. The choice $(\sqrt{73})$ avoids stability issues near the global minimum of $S$, while not damping iterations as much far away from it, by having Marquardt's parameter reducing $\mathbf{U}$ rapidly.

Equation (73) requires an estimate of $E\left[S\left(\mathbf{p}_{\mathrm{s}}\right)\right]$. The expectation of $S$ in (40) is [16]

$$
E\left[S\left(\mathbf{p}_{\mathrm{s}}\right)\right]=\operatorname{tr}(\operatorname{cov}(\boldsymbol{\omega}) \mathbf{W})+\sum_{j=1}^{n} \sum_{i} w_{i j} \operatorname{var}\left(\phi_{j}\left(x_{i}\right)\right),
$$

where $\operatorname{tr}$ is the trace operator. If $\mathbf{W}=(\operatorname{cov}(\boldsymbol{\omega}))^{-1}$ and $w_{i j}=\left(\operatorname{var}\left(\phi_{j}\left(x_{i}\right)\right)\right)^{-1}$ were chosen, 74 ) equals $n(1+m)$, where $m$ is the number of positions $x_{i}$ where mode shapes are measured. Typically measurement covariances are unknown, so $E\left[S\left(\mathbf{p}_{\mathrm{s}}\right)\right]=n(1+m)$ is an approximation.

We employed the convergence criteria

$$
S\left(\hat{\mathbf{p}}_{\mathrm{s}, k}\right)<\kappa E\left[S\left(\mathbf{p}_{\mathrm{s}}\right)\right],\left|\frac{\Delta \hat{p}}{\hat{p}+\delta_{1}}\right|<\delta_{2},\left|\frac{\Delta \hat{k}_{1-4}}{\hat{k}_{1-4}+\delta_{1}}\right|<\delta_{2},
$$

where $\Delta \hat{p}$ and $\Delta \hat{k}_{1-4}$ denotes corrections of parameters using 43 , and suggested convergence parameters are $\delta_{1}=10^{-6}$, and $\delta_{2}=10^{-3}$.

Sometimes iterations converge to a local minimum, but it is unknown whether this minimum is local or global. If $S>\kappa E\left[S\left(\mathbf{p}_{\mathrm{s}}\right)\right]$, we suspect the minimum is not global, but still the iteration procedure will get stuck, as $h_{k}$ in (46) becomes very small. For an iteration, if $h_{k}<0.01$ and $S>\kappa E\left[S\left(\mathbf{p}_{\mathrm{s}}\right)\right]$, we employed an additional updating rule

$$
\hat{\mathbf{p}}_{\mathrm{s}, k+1}=\operatorname{diag}\left(10^{r_{1}}, \ldots 10^{r_{5}}\right) \hat{\mathbf{p}}_{\mathrm{s}, k}
$$

where $r_{1-5}$ are independent random numbers between $-1 / 4$ and 1/4. Updating via (76) randomly updates $\hat{\mathbf{p}}_{\mathrm{s}, k+1}$ near local minimums, so that iterations can escape these.

\subsubsection{Re. Step 7, dealing with small, large, and negative parameters}

An issue arises using (43), as an updated set of parameters $\hat{\mathbf{p}}_{\mathrm{s}, k+1}$ may have negative, very large, or very small elements. Negative parameters could imply negative modal stiffness, with $\omega_{j}$ not representing a real-valued natural frequency. Very large or small boundary stiffness parameters, or very small tension has been found to cause $\mathbf{H}$ to be singular, because natural frequencies and mode shapes are insensitive to changes in parameters in these ranges. Very large tension reduces numerical precision when computing the hyperbolic terms of $\hat{\phi}_{j}(x)$. Because of these complications, estimation in this work is restricted to positive boundary parameters, and tension that are numerically neither very small or very large. A constraint condition will be formulated. To restrict parameters within certain limits, (43) is modified as follows:

First, low and high limits $10^{-8}$ and $10^{4}$ are set for all parameters in $\hat{\mathbf{p}}_{\mathrm{s}}$. If any parameter cross a limit after an update $43, \hat{\mathbf{p}}_{\mathrm{s}, k+1}$ is split into $r$ parameters $\hat{\mathbf{p}}_{\mathrm{s}, k+1}^{(2)} \in \mathbb{R}^{r}$ crossing a limit and remaining parameters $\hat{\mathbf{p}}_{\mathrm{s}, k+1}^{(1)} \in \mathbb{R}^{5-r}$. Crossing parameters $\hat{\mathbf{p}}_{\mathrm{s}, k+1}^{(2)}$ are suggested updated by

$$
\hat{\mathbf{p}}_{\mathrm{s}, k+1}^{(2)}=\hat{\mathbf{p}}_{\mathrm{s}, k}^{(2)}+\frac{h_{k}^{*}}{2}\left(\mathbf{p}_{1}-\hat{\mathbf{p}}_{\mathrm{s}, k}^{(2)}\right),
$$


where $\mathbf{p}_{1} \in \mathbb{R}^{r}$ is a vector of limits that would have otherwise been crossed, elements are either $10^{-8}$ or $10^{4}$, so parameters that would have otherwise crossed a limit are now a weighted average of their previous value and the limit. Noncrossing parameters are suggested updated as

$$
\hat{\mathbf{p}}_{\mathrm{s}, k+1}^{(1)}=\hat{\mathbf{p}}_{\mathrm{s}, k}^{(1)}+h_{k}^{*} \mathbf{H}^{(1)}\left(\hat{\mathbf{p}}_{\mathrm{s}, k}\right)^{-1} \mathbf{h}^{(1)}\left(\hat{\mathbf{p}}_{\mathrm{s}, k}\right),
$$

where $\mathbf{H}^{(1)} \in \mathbb{R}^{(5-r) \times(5-r)}$ and $\mathbf{h}^{(1)} \in \mathbb{R}^{5-r}$ are given by $69-(70)$, but with removed rows and columns associated with limit-crossing parameters. A new Box-Kanemasu's coefficient $h_{k}^{*}$ should be chosen. Relation (46) is still used, but (47) is replaced by

$$
a_{1}=-2 \mathbf{h}\left(\hat{\mathbf{p}}_{\mathrm{s}, k}\right)^{\mathrm{T}}\left(\left.\hat{\mathbf{p}}_{\mathrm{s}, k+1}\right|_{h_{k}^{*}=1}-\hat{\mathbf{p}}_{\mathrm{s}, k}\right), a_{2}=S\left(\left.\hat{\mathbf{p}}_{\mathrm{s}, k+1}\right|_{h_{k}^{*}=1}\right)-S\left(\hat{\mathbf{p}}_{\mathrm{s}, k}\right)-a_{1},
$$

where $\left.\hat{\mathbf{p}}_{\mathrm{s}, k+1}\right|_{h_{k}^{*}=1}$ is the updated parameter estimate from $777-78$ if $h_{k}^{*}$ was unity.

\subsection{Summary}

This section suggested a Gauss-Newton iteration method for fitting unknown tension and boundary stiffness parameters to an undamped beam model given measured natural frequencies and/or mode shapes. Next Methods 1 and 2 are validated for illustrative example cases.

\section{Validation}

Here natural frequencies, mode shapes and damping coefficients are simulated for a beam with nondimensional tension $p=100$, boundary stiffness parameters $k_{1}=1000, k_{2}=0.001, k_{3}=$ $1000, k_{4}=0.001$ and boundary damping parameters $c_{1}=1, c_{2}=0.1, c_{3}=1, c_{4}=0.1$, unless otherwise stated. Calculated properties are used in the following sections to estimate boundary parameters and tension, Method 2 have been used on experimental data as well, cf. Section 5.4.

\subsection{Stiffness estimation using Method 1, simulated data}

Figure 3 shows tension and boundary stiffness estimation results $\hat{p}$ and $\hat{k}_{1-4}$, using Method 1. Five measured natural frequencies and mode shapes are simulated. Rotational stiffness $k_{3}$ at the beams left boundary is varied. Weights used in (37) are chosen as $\mathbf{U}_{\mathrm{s}}=\mathbf{0}$, and $\mathbf{W}_{\mathrm{s}}=$ $\operatorname{diag}\left(\omega_{1}^{-4}, \ldots \omega_{5}^{-4}\right)$ to weigh relative errors equally between frequencies.

Figure 3 shows true and estimated parameters through (37), where $k_{1}$ and $p$ are observed to be estimated correctly for all $k_{3}$. As discussed with Figure 2 , very small or very large boundary stiffness parameters cannot be estimated accurately from natural frequencies; this is the case for too small $k_{2}=10^{-3}$ and $k_{4}=10^{-3}$ in Figure 3 , while $k_{3}$ is too small below $k_{3}=10^{-3}$ and too large above $k_{3}=10^{6}$. Parameters in these ranges do not affect natural frequencies, so are unimportant for producing correct natural frequencies. If a stiffness is estimated to be very large or very small, it can be considered infinite or zero respectively, and similarly with small tension.

Method 1 has been used on other cases, where no parameters are very large or very small, resulting in accurate estimates. Method 1 is validated in the sense that for all cases tested, it estimates parameters that correctly predict natural frequencies, when true continuous mode shapes are known. 

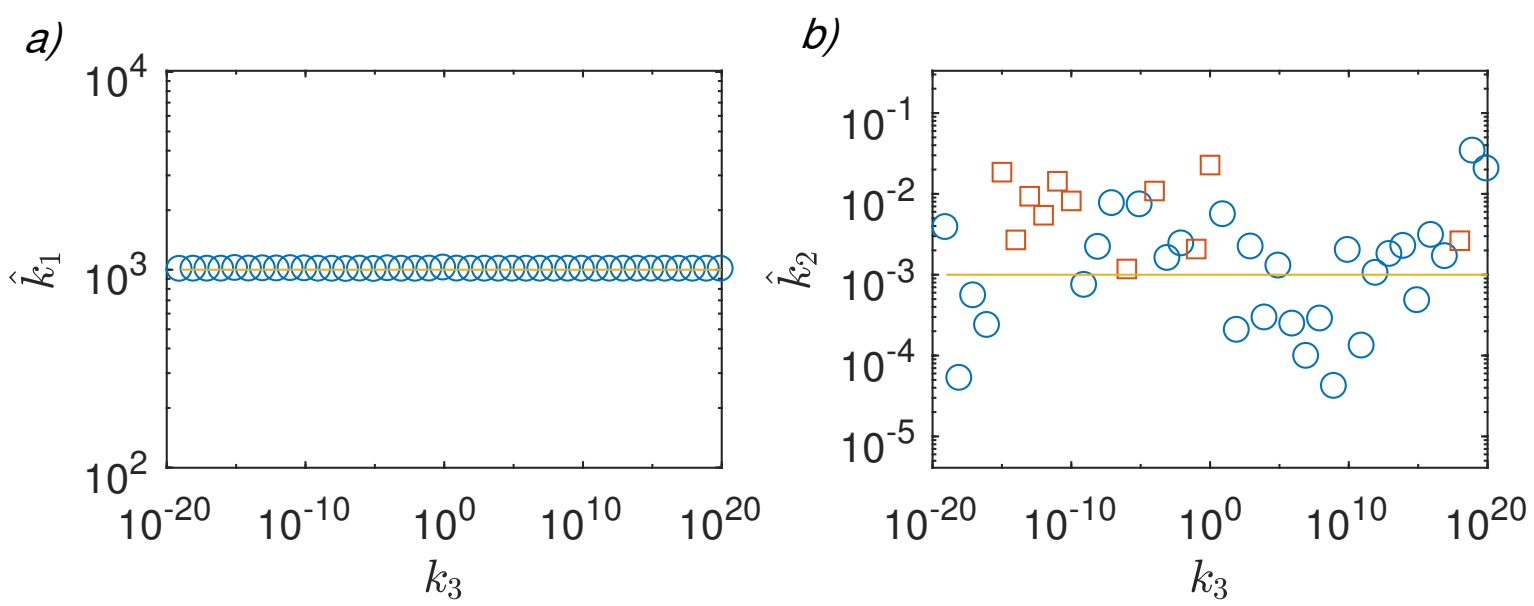

c)

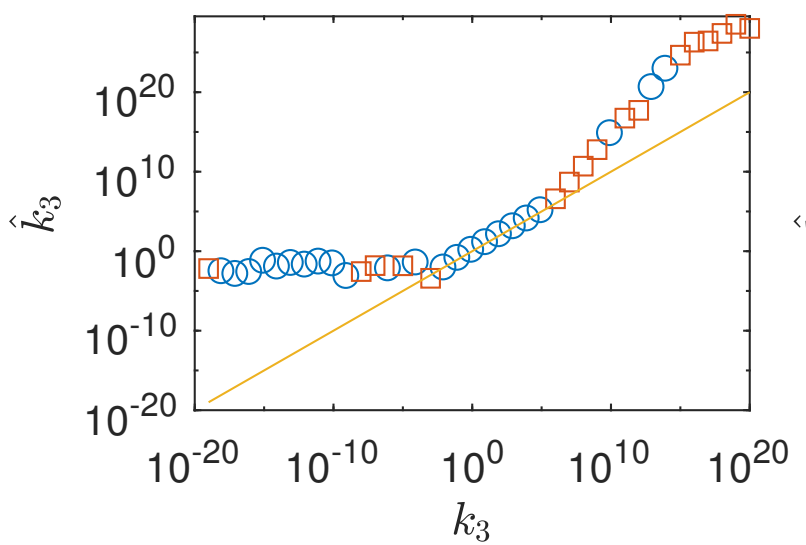

e)

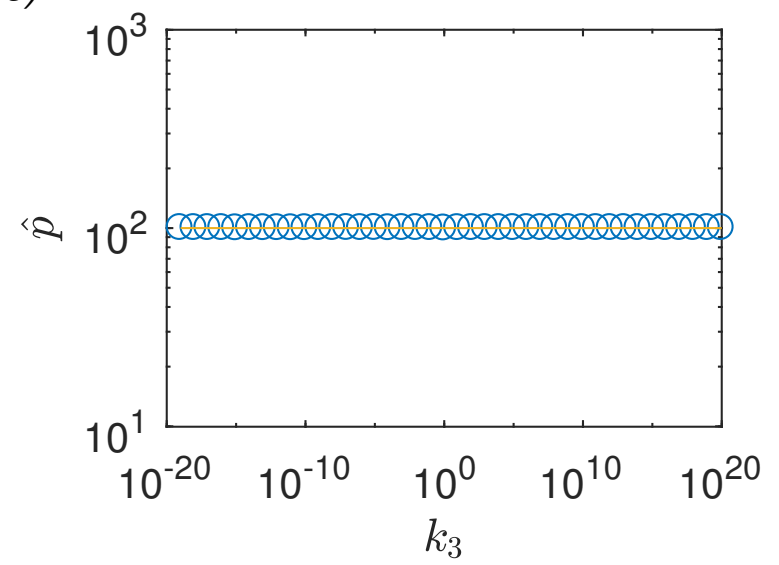

d)

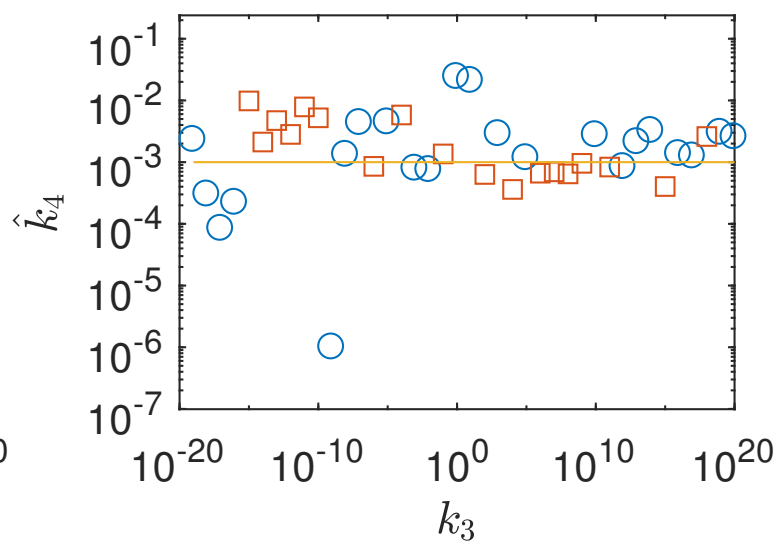




\subsection{Stiffness estimation using Method 2, simulated data}

Figure 4 shows iterations of tension and boundary stiffness estimates using (43). Eight measured natural frequencies are simulated. The weights in 40 are chosen as $\mathbf{W}=\sigma_{\omega}^{-2} \operatorname{diag}\left(\omega_{1}^{-2}, \ldots \omega_{8}^{-2}\right)$, and $w_{j}(x)=0$ since mode shapes are not simulated. The value $\sigma_{\omega}=10^{-8}$ reflects numerical precision in calculating $\omega_{j}$ 's. The initial parameter estimate used is $\hat{\mathbf{p}}_{\mathrm{s}, 0}=\left\{\begin{array}{lllll}1 & 2 & 1 & 2 & 3\end{array}\right\}^{\mathrm{T}}$, chosen to have no large or small entries, and no identical boundaries.

For the first iterations, parameter estimates do not update significantly, but then starts converging. The slow updating for the first iterations is due to large confidence $\mathbf{U}$ in a posteriori estimate $\tilde{\mathbf{p}}_{s}$. As Marquardt's parameter $\lambda_{\mathrm{m}}$ in (73) decreases, $\mathbf{U}$ decreases so measurements are trusted more in each iteration.

Boundary stiffness parameters converges to swapped boundaries $\hat{k}_{1}=k_{2}, \hat{k}_{2}=k_{1}, \hat{k}_{3}=k_{4}, \hat{k}_{4}=$ $k_{3}$. This happens by coincidence - with another choice of initial estimate $\hat{\mathbf{p}}_{\mathrm{s}, 0}$, boundary stiffness parameters would converge to true values, i.e. unswapped. Boundary stiffness parameters can converge to swapped boundary values because natural frequencies cannot distinguish left and right boundaries.

The iteration (43) shown in Figure 4 is repeated for a case where uncertainty corresponding to measurement variability is added to natural frequencies, and to a case where mode shapes at eleven equally spaced points, $x_{i}=0,0.1, \ldots 1$, on the beam have been simulated. In the former case, uncertainty is added by multiplying $\omega_{j}$ with a number picked from a normal distribution $\mathrm{N}\left(1, \sigma_{\omega}^{2}\right)$ with $\sigma_{\omega}=10^{-2}$. In the latter case, simulated mode shapes are normalized with $\int_{0}^{1} \phi_{j}(x)^{2} d x=1$ and uncertainty from a normal distribution $\mathrm{N}\left(0, \sigma_{\phi}^{2}\right)$ with $\sigma_{\phi}=0.1$ is added to each simulated mode shape measurement. This uncertainty makes optimal $w_{i j}=\sigma_{\phi}^{-2}$.

Table 1 shows estimated parameters from ten average Monte Carlo procedures [16]. Only ten averages are used due to CPU time. The notation $\hat{k}_{2}=3.0 \pm 3.2$ means the average $\hat{k}_{2}$ is 3.0, while its standard deviation is 3.2 , but since $\hat{k}_{2}$ obtained from Monte Carlo simulations has a skewed probability distribution, $\hat{k}_{2}=3.0 \pm 3.2$ does not mean $\hat{k}_{2}$ is sometimes estimated below zero.

Table 1 shows reduced precision on measurements reduces estimation precision. Without mode shape data, estimated boundaries may be swapped. Method 2 is validated in the sense that it, for all cases studied, estimates parameters that correctly predict measured data, and will give accurate results when measured data are sensitive to parameters.

Table 1. Tension and boundary stiffness estimates and std. using (43)

\begin{tabular}{lrrrr}
\hline$\hat{\mathbf{p}}_{s}$ & True & $\sigma_{\omega}=10^{-8}$ & $\sigma_{\omega}=10^{-2}$ & $\sigma_{\omega}=10^{-2}, \sigma_{\phi}=0.1$ \\
\hline$\hat{p}$ & 100 & $100 \pm 0$ & $99.2 \pm 4.9$ & $97.0 \pm 5$ \\
$\hat{k}_{1}$ & 1000 & $0.001 \pm 0$ & $3.0 \pm 3.2$ & $992 \pm 61.9$ \\
$\hat{k}_{2}$ & 0.001 & $1000 \pm 0$ & $1020 \pm 82.5$ & $5.2 \pm 5.1$ \\
$\hat{k}_{3}$ & 1000 & $0.001 \pm 0$ & $1.6 \pm 4.3$ & $1310 \pm 2930$ \\
$\hat{k}_{4}$ & 0.001 & $1000 \pm 0$ & $1260 \pm 2680$ & $0.7 \pm 0.55$ \\
\hline
\end{tabular}


a)

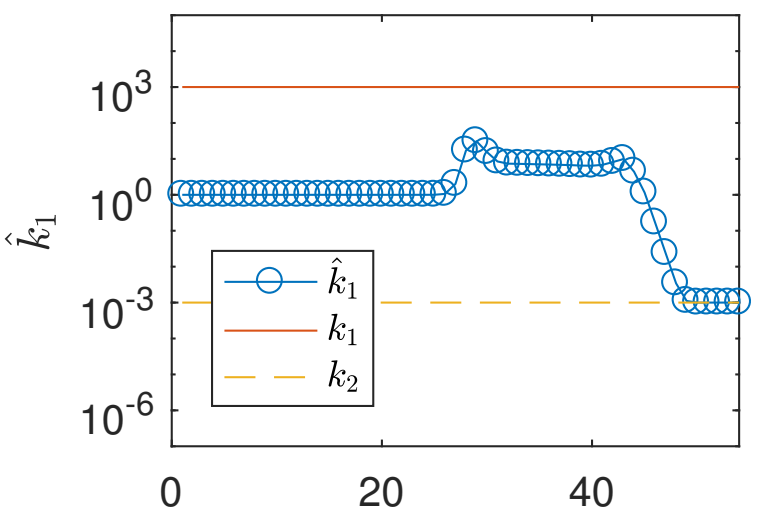

c)

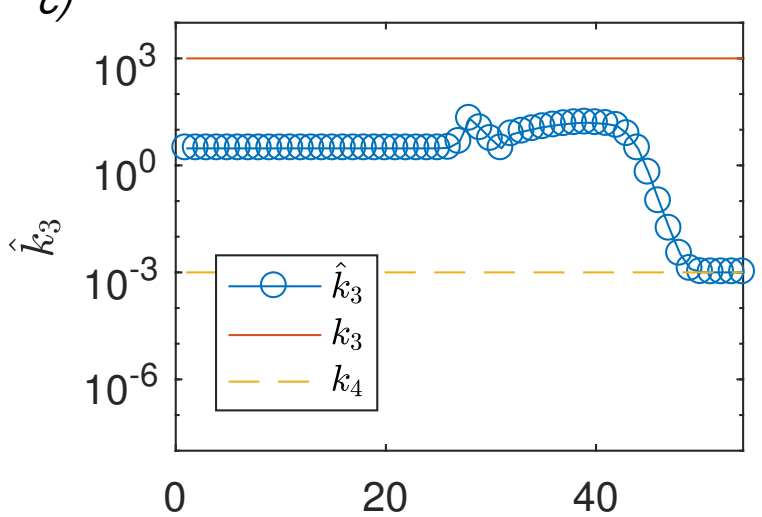

e)

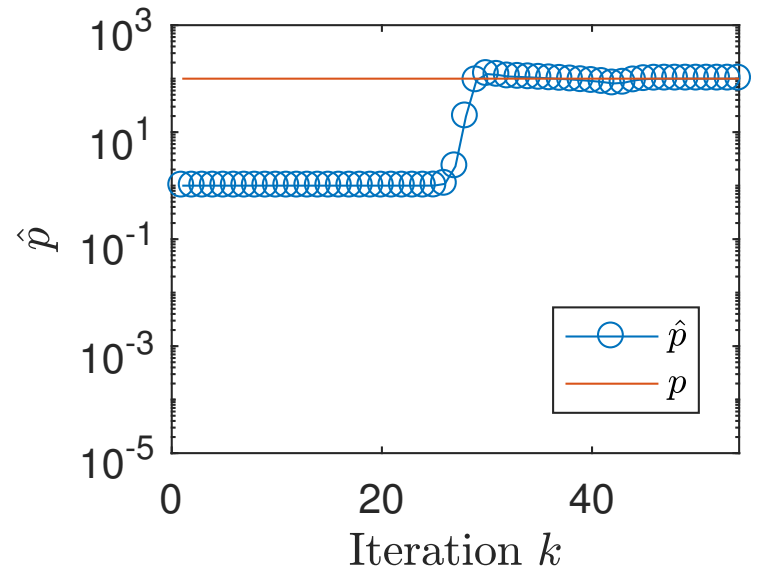

b)

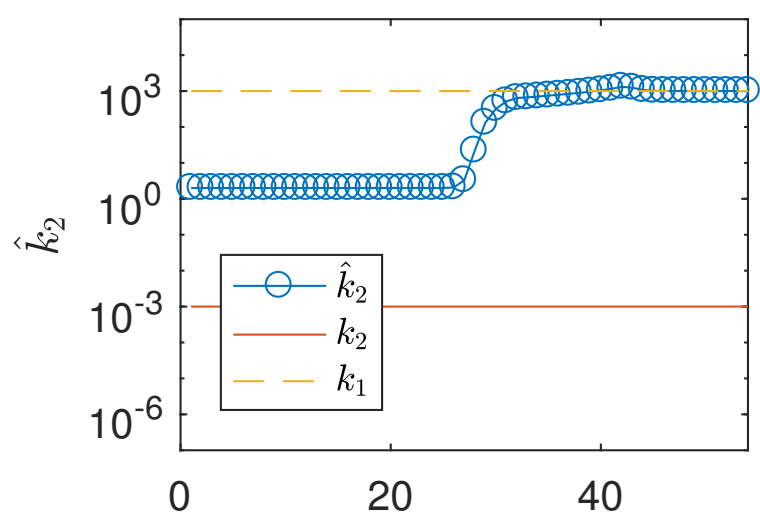

d)

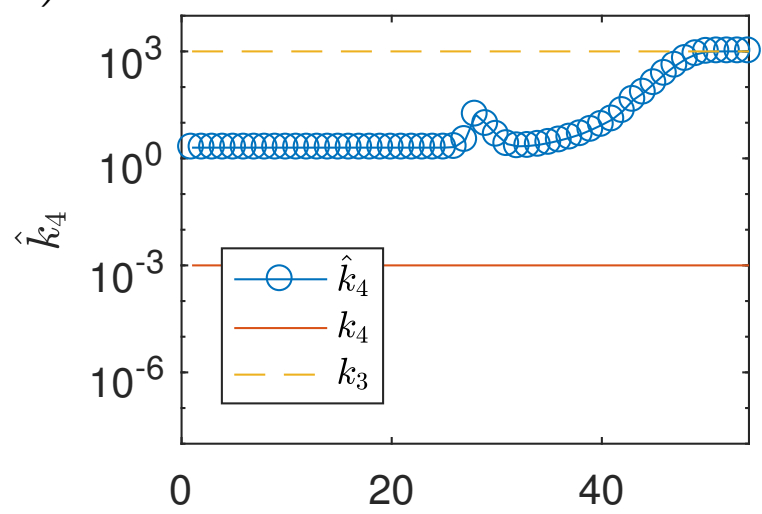

Fig. 4. Estimates a) $\hat{k}_{1}$, b) $\hat{k}_{2}$, c), $\hat{k}_{3}$, d), $\hat{k}_{4}$ and e) $\hat{p}$ of parameters based on 43 using simulated natural frequencies, with true values $p=10^{2}, k_{1}=10^{3}, k_{2}=10^{-3}, k_{3}=10^{3}$, and $k_{4}=10^{-3}$. 


\subsection{Boundary damping estimation using Method 1 and 2 combined, simulated data}

Next we test Method 1 for estimating boundary damping, when true damping parameters are $c_{1}=1, c_{2}=0.1, c_{3}=1, c_{4}=0.1$ and there is no beam damping forces, $\mathrm{D}=0$. We simulate a real-world application where only natural frequencies and damping coefficients of eight modes are known with uncertainty, but mode shapes are unknown. Boundary damping cannot be estimated with (38) without knowledge of mode shapes, so we will employ a trick to obtain them. Using Method 2 with eight simulated measured natural frequencies with uncertainty (cf. Section 5.2), estimated stiffness parameters are found as $\hat{\mathbf{p}}_{\mathrm{s}}=\left\{\begin{array}{llllll}99.2 & 1020 & 3.0 & 1260 & 1.6\end{array}\right\}^{\mathrm{T}}$ from the fourth column of Table 1 (but without swapped boundaries). These stiffness parameters can then be used to calculate undamped mode shapes, which are assumed close in absolute value to damped mode shapes, because damping is small.

Thus mode shapes have been estimated using simulated measured natural frequencies, so boundary damping estimation using Method 1 can be employed.

Simulated measured damping coefficients $2 \zeta_{j} \omega_{j}, j=1,2 \ldots 8$, are calculated using (19), with true mode shapes, giving damping ratios in the range $0.8 \%$ to $16 \%$, and uncertainty is included by multiplying damping coefficients with a number from a normal distribution $\mathrm{N}\left(1, \sigma_{\mathrm{d}}^{2}\right)$. Damping coefficient uncertainties, $\sigma_{\mathrm{d}}$, simulate measurement noise. The optimal weights for (36) are then $\mathbf{U}_{\mathrm{c}}=\mathbf{0}$, and $\mathbf{W}_{\mathrm{c}}=(\operatorname{cov}(\mathbf{d}))^{-1}=\sigma_{\mathrm{d}}^{-2} \operatorname{diag}\left(\left(2 \zeta_{1} \omega_{1}\right)^{-2}, \ldots\left(2 \zeta_{8} \omega_{8}\right)^{-2}\right)$, but since a scaling of $\mathbf{W}_{\mathrm{c}}$ does not change the estimate $\sqrt{38}), \sigma_{\mathrm{d}}^{-2}$ is omitted choosing instead $\mathbf{W}_{\mathrm{c}}=\operatorname{diag}\left(\left(2 \zeta_{1} \omega_{1}\right)^{-2}, \ldots\left(2 \zeta_{8} \omega_{8}\right)^{-2}\right)$.

Two simulated experiments are carried out, with different damping coefficient uncertainties $\sigma_{\mathrm{d}}$. Table 2 shows estimation results from a hundred Monte Carlo averages [16] of [38].

Table 2. Damping coefficient uncertainty, boundary damping estimates and std.

\begin{tabular}{lrrrr}
\hline & $\mathbf{p}_{\mathrm{c}}$ & $\hat{\mathbf{p}}_{\mathrm{c}}$ & $\hat{\mathbf{p}}_{\mathrm{c}}$ & Std. from $\operatorname{cov}\left(\hat{\mathbf{p}}_{\mathrm{c}}\right)$ in $\sqrt{39}$ \\
\hline$\sigma_{d}$ & & 0 & 0.1 & 0.1 \\
$c_{1}$ & 1 & 1.25 & $1.27 \pm 0.279$ & \pm 0.254 \\
$c_{2}$ & 0.1 & 0.105 & $0.103 \pm 0.019$ & \pm 0.020 \\
$c_{3}$ & 1 & -46.1 & $-39.2 \pm 78.7$ & \pm 78.2 \\
$c_{4}$ & 0.1 & 0.125 & $0.124 \pm 0.010$ & \pm 0.010 \\
\hline
\end{tabular}

Estimate $\hat{c}_{2}$ is accurate, $\hat{c}_{1}$ and $\hat{c}_{4}$ are within an order of magnitude and $\hat{c}_{3}$ is inaccurate. Parameter $c_{3}$ damps movements that are restricted by large stiffness $k_{3}=10^{3}$ and $c_{2}$ damps movements that are unrestricted due to small stiffness $k_{2}=10^{-3}$. Thus $c_{3}$ must be insignificant on damping coefficients and $c_{2}$ must be significant, explaining why $\hat{c}_{2}$ is accurate and $\hat{c}_{3}$ is not.

The estimation error due to using estimated and not true mode shapes in $\mathbf{B}_{\mathrm{c}}$ causes errors, as simulation with $\sigma_{\mathrm{d}}=0$ still estimates wrong damping parameters.

The case analyzed is one of many where Method 1 has been applied for boundary damping estimation. The results are similar to what is observed from Table 2, i.e. boundary damping estimations are accurate, when a damping parameter significantly affects damping coefficient, and inaccurate when it does not.

But if boundary damping parameters are not known, which is the point of estimating them, how can it be known which boundary damping parameters are and are not significant? We sug- 
gest is using an assumed damping coefficient uncertainty model to calculate estimation standard deviation through (39), as the square roots of the diagonal elements in $\operatorname{cov}\left(\hat{\mathbf{p}}_{\mathrm{c}}\right)$ with $\operatorname{cov}(\mathbf{d})=$ $\sigma_{\mathrm{d}}^{2} \operatorname{diag}\left(\left(2 \zeta_{1} \omega_{1}\right)^{2}, \ldots\left(2 \zeta_{8} \omega_{8}\right)^{2}\right)$. This is shown in the last column of Table 2 , revealing $\hat{c}_{3}$ to be an uncertain estimate.

\subsection{Free-free steel beam, experimental data}

Method 2 is here used with natural frequencies from a steel beam suspended in compliant rubber bands. This case is chosen because experimental data was at hand [21], and all stiffness parameters are approximately known. There is no axial tension, $p=0$, and rubber bands supporting the beam are so compliant their normalized boundary stiffness $k_{1-4}$ can be considered close to zero. The left column of Table 3 shows natural frequency data nondimensionalized by $\omega_{0}$. Two rigid body natural frequencies are set to zero.

Table 3. Nondimensional measured and estimated natural frequencies

\begin{tabular}{rr}
\hline$\omega$ & $\hat{\omega}$ \\
\hline 0 & 0.6 \\
0 & 1.1 \\
22.4 & 22.4 \\
61.7 & 61.7 \\
120.9 & 120.9 \\
199.3 & 199.9 \\
297.0 & 298.6 \\
414.0 & 417.0 \\
549.9 & 555.2 \\
704.5 & 713.1 \\
877.4 & 890.7 \\
1068.2 & 1088.1 \\
\hline
\end{tabular}

Table 4. Estimated stiffness parameters, freefree steel beam

\begin{tabular}{ll}
\hline & $\hat{\mathbf{p}}_{\mathrm{s}}$ \\
\hline$\hat{p}$ & $10^{-8}$ \\
$\hat{k}_{1}$ & 0.199 \\
$\hat{k}_{2}$ & 0.199 \\
$\hat{k}_{3}$ & $10^{-8}$ \\
$\hat{k}_{4}$ & $10^{-8}$ \\
\hline
\end{tabular}

For choosing $\mathbf{W}$ we used the uncertainty model:

$$
\sigma_{1}=\sigma_{2} \rightarrow \infty, \quad \sigma_{j}=\frac{1}{8} \Delta \omega+\frac{1}{100} \omega_{j}, j=3,4 \ldots 12
$$

where $\sigma_{j}$ is the standard deviation of natural frequencies $\omega_{j}, \Delta \omega$ is nondimensional frequency resolution, $\Delta \omega=0.65$, and $\omega_{j}$ is the $j$ 'th natural frequency. The uncertainties of rigid body natural frequencies, $\omega_{1}$ and $\omega_{2}$, are infinite, as they were not measured. The model (80) employs a fraction $1 / 8$ of the frequency resolution $\Delta \omega$, representing rounding deviations, and a fraction $1 / 100$ of $\omega_{j}$, representing relative errors caused by e.g. erroneous nondimensionalization. The uncertainty model $(80)$ represents assumed measurement noise. From 80$) \mathbf{W}=(\operatorname{cov}(\boldsymbol{\omega}))^{-1}=$ $\operatorname{diag}\left(0,0, \sigma_{3}^{-2}, \ldots \sigma_{12}^{-2}\right)$.

Parameter estimates are calculated using 43 , with initial estimate $\hat{\mathbf{p}}_{\mathrm{s}, 0}=\left\{\begin{array}{lllll}1 & 1 & 1 & 1 & 1\end{array}\right\}^{\mathrm{T}}$. Since the starting boundaries are identical and natural frequencies used for fitting cannot distinguish $k_{1}, k_{3}$ from $k_{2}, k_{4}$, iterations using (43) will result in identical corrections to each boundary. 
Thus, the final estimate $\hat{\mathbf{p}}_{\mathrm{s}}$ will have identical boundaries, which is intended, as the beam is expected to have identical boundaries.

Table 4 shows the results, agreeing with expected values of zero for $\hat{k}_{3}, \hat{k}_{4}$ and $\hat{p}$, as $10^{-8}$ is a coded lower estimation limit. Estimates $\hat{k}_{1}$, and $\hat{k}_{2}$ are small but not zero, and might represent finite stiffness of compliant rubber-bands supporting the beam.

The second column of Table 3 shows natural frequencies calculated from estimates in Table 4 , and are within $2 \%$ of measured natural frequencies.

Damping coefficients were also measured for the beam, and boundary damping estimation with Method 1 was attempted, but did not work well. Damping coefficients were not reproducible, which is believed to be due to dominating damping sources other than boundary damping, so (19) does not hold.

\subsection{Summary}

Methods 1 and 2 have been validated using simulated data in the sense that, for all cases tested, estimated parameters predict measured data correctly. This is a useful result for modeling purposes. If accurate estimates of boundary parameters and tension is sought for, Methods 1 and 2 will work unless boundary parameters are very large or very small, or the tension is very small. Method 2 has been validated using free-free beam experimental data, though boundary damping parameter estimation with Method 1 did not work well, believed to be due to damping from other sources than boundaries.

\section{Conclusion}

Two estimation methods have been developed to estimate boundary parameters and tension for elastic beams using measured natural frequencies, mode shapes, and damping coefficients. Method 1 applies linear regression to an expression for Rayleigh's quotient to estimate boundary stiffness parameters and tension, based on measured natural frequencies and mode shapes, and also estimates boundary damping parameters based on measured damping coefficients and mode shapes. Linear regression is an advantage due to simplicity and uniqueness of estimates, but disadvantageously in this case requires measured continuous mode shapes. Estimation using Rayleigh's quotient does not accurately estimate very large or very small parameters, since measured natural frequencies and damping coefficients are insensitive to changes in boundary parameters when boundary stiffness is very large or small and insensitive to changes in tension when tension is very small; however large parameters are estimated large and small parameters are estimated small.

Method 2 uses nonlinear regression with the eigenvalue problem formulation for an EulerBernoulli beam to estimate boundary stiffness parameters and tension; it is usable with measured natural frequencies, mode shapes, or both. Boundary stiffness parameters that are very small or large, and tension that is very small, are not accurately estimated with this method, since natural frequencies and mode shapes are insensitive to changes in boundaries in these ranges; however very large parameters are estimated large and very small parameters are estimated small. The method works even when boundaries are identical, when mode shapes are used for fitting, but has a disadvantage in requiring heavier computations than Method 1, and in not estimating boundary damping parameters. 


\section{References}

[1] J. J. Thomsen, Vibrations and Stability: Advanced Theory, Analysis and Tools, Springer-Verlag Berlin Heidelberg, 2003.

[3] J. J. Thomsen, S. M. Sah, A. Fidlin, D. Tcherniak, Vibration-based testing of bolted joints, in: 9 European Nonlinear Dynamics Conference (ENOC), 25-30 June Budapest, 2017.

[4] M. Kataoka, S. Ohno, E. Marui, Calculation of natural frequencies of bending vibration of straight rod with additional mass and subjected to axial compression, and estimation of axial force, JSME International Journal Series C 37 (1) (1994) 21-27.

[5] K. Maes, J. Peeters, E. Reynders, G. Lombaert, G. Roeck, Identification of axial forces in beam members by local vibration measurements, Journal of Sound and Vibration 332 (21) (2013) 5417-5432

[6] S. Chaki, G. Corneloup, I. Lillamand, H. Walaszek, Combination of longitudinal and transverse ultrasonic waves for in situ control of the tightening of bolts, Journal of Pressure Vessel Technology - Transactions of the Asme 129 (3) (2007) 383-390

[7] A. Dick, B. Balachandran, D. DeVoe, C. Mote Jr., Parametric identification of piezoelectric microscale resonators, Journal of Micromechanics and Microengineering 16 (2006) 1593-1601

[8] G. Rebecchi, N. Tullini, F. Laudiero, Estimate of the axial force in slender beams with unknown boundary conditions using one flexural mode shape, Journal of Sound and Vibration 332 (18) (2013) 4122-4135

[9] J. A. Inaudi, M. Kelly, Linear Hysteretic Damping and the Hilbert Transform, Journal of Engineering Mechanics 121 (5) (1995) 626-632

[10] L. Ljung, System Identification: Theory for the User, Prentice-Hall, Englewood Cliffs, N. J., 1987.

[11] J. S. Bendat, P. A. Palo, R. N. Coppolino, A general identification technique for nonlinear differential equations of motion, Probabilistic Engineering Mechanics 7 (1992) 43-61

[12] J. S. Bendat, R. N. Coppolino, P. A. Palo, Identification of Physical Parameters with Memory in Non-linear Systems, International Journal of Non-Linear Mechanics, 30 (6) (1995) 841-860

[13] S. F. Masri, T. K. Caughey, A nonparametric identification technique for nonlinear dynamic problems, Journal of Applied Mechanics 46 (1979) 433-447

[14] K. Worden, J. R. Wright, M. A. Al-Hadid, Experimental Identification of multi degree-of-freedom nonlinear systems using restoring force methods, International Journal of Analytical and Experimental Modal Analysis 9 (1) (1994) 35-55

[15] T. B. Schön, Estimation of Nonlinear Dynamic Systems Theory and Applications, Linköping Studies in Science and Technology. Dissertations 998 (2006)

[16] J. Beck, K. Arnold, Parameter Estimation in Engineering and Science, John Wiley \& Sons, 1977.

[17] J. J. Thomsen, Modelling Human Tibia Structural Vibrations, Journal of Biomechanics 23 (3) (1990) 215-228 
[18] M. Hashimoto, E. Marui, S. Kato, Estimation of contact stiffness at interfaces in machine structures by a beam model on an elastic foundation, Tribology International 27 (6) (1994) 423-431

[19] M. D. Todd, J. M. Nichols, C. J. Nichols, L. N. Virgin, An assessment of modal property effectiveness in detecting bolted joint degradation: theory and experiment, Journal of Sound and Vibration 275 (2004) 1113-1126

[20] A. S. Phani, S. Adhikari, Rayleigh quotient and dissipative systems, Journal of Applied Mechanics 75 (2008).

[21] [dataset] M. Brøns, Technical University of Denmark, Department of Mechanical Engineering, Personal communications, 2017. 